\title{
TWO-PHASE FLOW OVER FLOODED MICRO-PILLAR STRUCTURES WITH ENGINEERED WETTABILITY
}

\author{
A Thesis presented to the Faculty of the Graduate School \\ at the University of Missouri-Columbia
}

In Partial Fulfillment

Of the Requirements for the Degree

Master of Science

by

ZHOU ZHOU

Dr.Chung-Lung Chen, Thesis Supervisor

DECEMBER 2013 
The undersigned, appointed by the Dean of the Graduate School, have examined the thesis entitled

\section{TWO-PHASE FLOW OVER FLOODED MICRO-PILLAR STRUCTURES WITH ENGINEERED WETTABILITY}

Presented by Zhou Zhou

A candidate for the degree of Master of Science

And hereby certify that, in their opinion, it is worthy of acceptance.

Professor Chung-Lung Chen

Professor Yuwen Zhang

Professor Stephen Montgomery-Smith 


\section{ACKNOWLEDGEMENTS}

First and foremost I would like to extend my deepest gratitude to my supervisor Dr. ChungLung Chen for having guided me through the field of high heat flux electronic cooling with your patience and knowledge. Also thank you to your startup funding for supporting this research and supporting me to study.

I would like to thank all of my colleagues in the Mechanical and Aerospace Engineering Department for your support. I would like to thank Simon Chen for his help in the lab and for always listening to my ideas. Sean Shi, thank you for all your help in the lab. I would also like to thank Steven Schafer for always being there and for thoroughgoing proofreading of my thesis.

Thank you to Pengfei, Huibin, Milad, Hayder, Baffoe, Jingwen, Shuyi and all my friends for making this graduate experience enjoyable. Thank you also to Prof. Yuwen Zhang and Prof. Stephen Montgomery-Smith for being part of my thesis committee and for taking part of your time to improve this work.

Finally, I sincerely appreciate my family for their support and encouragement throughout my graduate studies. I am grateful for your endless love. Special thanks to my girlfriend, Qiuhong Wang, you greatly inspired and motivated me. Thank you for being my support system for the last 2 years.

Zhou Zhou 


\section{TABLE OF CONTENTS}



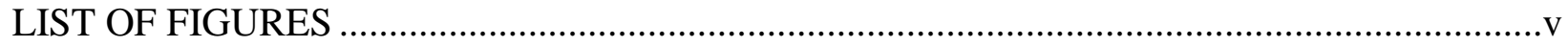

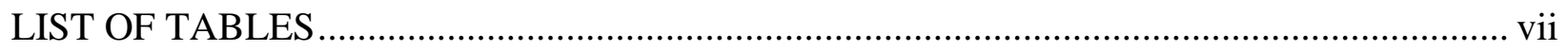

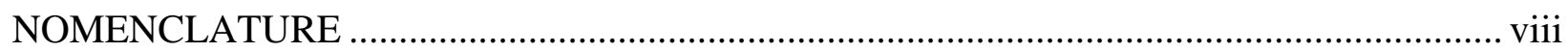

ABSTRACT ……............................................................................................................

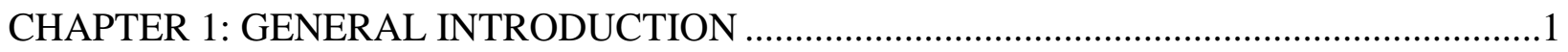

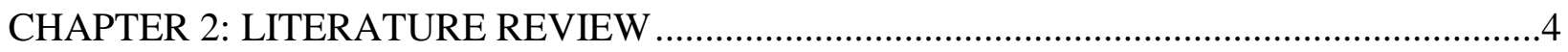

2.1 Droplet-based Thermal Management ………………................................................. 4

2.1.1 Electrowetting Thermal Management........................................................ 4

2.1.2 Spray Cooling Thermal Management................................................... 7

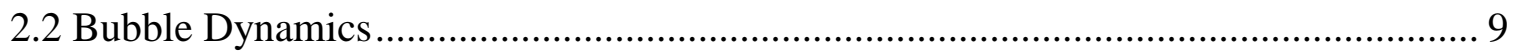

2.3 Phase Change Investigation with VOF Method............................................................ 11

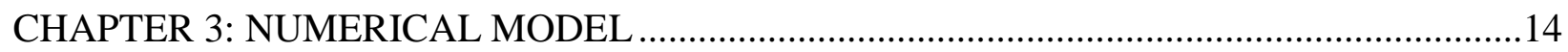

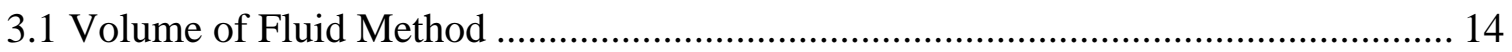

3.1.1 Phase Conservation .......................................................................................... 14

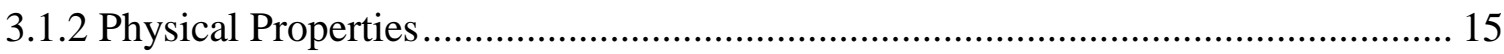

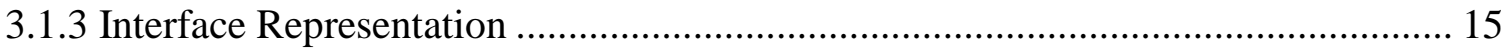

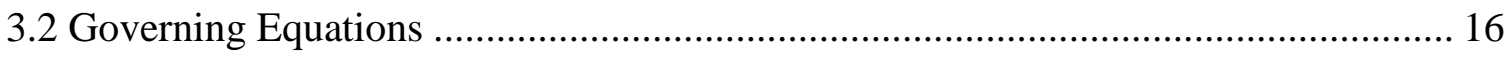







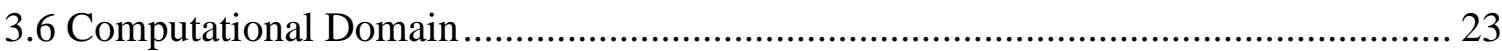


3.6.2 Microstructure topologies ............................................................... 24

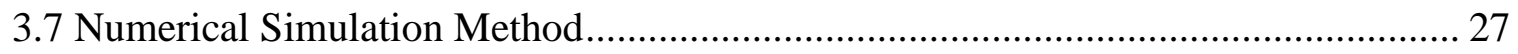



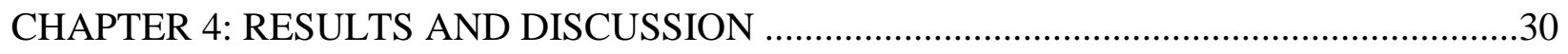

4.1 Structure and Wettability Effect on Bubble Dynamic Behavior .............................. 30

4.2 Comparative Studies for Structures with Vary Pillars Height ................................ 37

4.2.1 Constant Temperature Boundary Condition ......................................... 37

4.2.2 Constant Heat Flux Boundary Condition............................................... 40

4.3 Comparative Studies for Structures with Various Pillar Array Density ................... 46

4.3.1 Constant Temperature Boundary Condition ......................................... 46

4.3.2 Constant Heat Flux Boundary Condition............................................ 48

4.4 Comparative Study for Structures with Same Extra Solid-Liquid Interfacial Area ... 52

4.4.1 Constant Temperature Boundary Condition ...................................... 52

4.4.2 Constant Heat Flux Boundary Condition............................................. 54

4.5 Comparative Studies for Structures with Wettability Texture and Hydrophilic

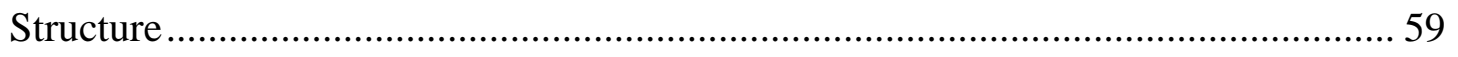



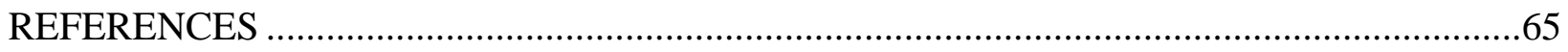




\section{LIST OF FIGURES}

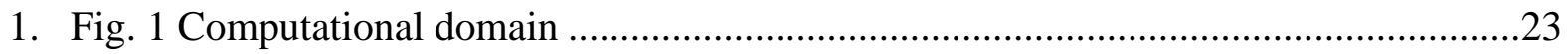



3. Fig. 3 Grid independent test for computational domain ..........................................29

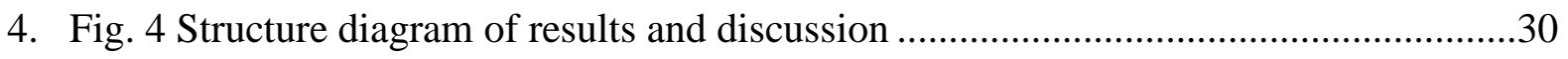

5. Fig. 5 Boiling phenomena on hydrophilic flat surface ............................................. 31

6. Fig. 6 Boiling phenomena on hydrophobic spot patterned flat surface ............................32

7. Fig. 7 Velocity field during bubble departure process on wettability patterned flat surface H0A55_9

8. Fig. 8 Boiling phenomena on hydrophilic structured surface ........................................34

9. Fig. 9 Boiling phenomena on micro-structured surface with wettability gradient ............35

10. Fig. 10 Velocity field during bubble departure process on structured surface H5A55_9

11. Fig. 11 Liquid temperature on surfaces with different pillar height $\left(\mathrm{T}_{\mathrm{w} \text {-bottom }}=400 \mathrm{~K}\right) \quad \ldots 38$

12. Fig. 12 Mass transfer rate on surfaces with different pillar height $\left(\mathrm{T}_{\mathrm{w}-\mathrm{bottom}}=400 \mathrm{~K}\right) \ldots \ldots .39$

13. Fig. 13 Temperature contours on surfaces with different pillar height $\left(\mathrm{q}_{\mathrm{w}}{ }^{\prime}=700 \mathrm{~W} / \mathrm{cm}^{2}\right)$

14. Fig. 14 Bottom surface temperature on surfaces with different pillar height $\left(\mathrm{q}_{\mathrm{w}} "=\right.$ $700 \mathrm{~W} / \mathrm{cm}^{2}$ )

15. Fig. 15 Mass transfer rate on surfaces with different pillar height $\left(\mathrm{q}_{\mathrm{w}}{ }^{\prime \prime}=700 \mathrm{~W} / \mathrm{cm}^{2}\right) \ldots . .44$

16. Fig. 16 Heat transfer coefficient on surfaces with different pillar height $\left(\mathrm{q}_{\mathrm{w}}{ }^{\prime}=\right.$ $\left.700 \mathrm{~W} / \mathrm{cm}^{2}\right)$ 
17. Fig. 17 Liquid temperature on surfaces with different pillar array density $\left(\mathrm{T}_{\mathrm{w} \text {-bottom }}=\right.$ $400 \mathrm{~K})$

18. Fig. 18 Mass transfer rate on surfaces with different pillar array density $\left(\mathrm{T}_{\mathrm{w} \text {-bottom }}=400 \mathrm{~K}\right)$

19. Fig. 19 Temperature contours on surfaces with different pillar array density $\left(\mathrm{q}_{\mathrm{w}}{ }^{\prime}=\right.$ $\left.700 \mathrm{~W} / \mathrm{cm}^{2}\right)$

20. Fig. 20 Bottom surface temperature on surfaces with different pillar array density $\left(q_{w} "=\right.$ $\left.700 \mathrm{~W} / \mathrm{cm}^{2}\right)$

21. Fig. 21 Mass transfer rate on surfaces with different pillar array density $\left(\mathrm{q}_{\mathrm{w}}{ }^{\prime \prime}=\right.$ $\left.700 \mathrm{~W} / \mathrm{cm}^{2}\right)$

22. Fig. 22 Heat transfer coefficient on surfaces with different pillar array density $\left(\mathrm{q}_{\mathrm{w}}{ }^{\prime}=\right.$ $\left.700 \mathrm{~W} / \mathrm{cm}^{2}\right)$

23. Fig. 23 liquid temperature on surfaces for comparison of impact weight between pillar height and pillar array density $\left(\mathrm{T}_{\mathrm{w}-\text { bottom }}=400 \mathrm{~K}\right)$

24. Fig. 24 Mass transfer rate on surfaces for comparison of impact weight between pillar height and pillar array density $\left(\mathrm{T}_{\mathrm{w}-\text { bottom }}=400 \mathrm{~K}\right)$

25. Fig. 25 Bottom surface temperature on surfaces for comparison of impact weight between pillar height and pillar array density $\left(\mathrm{q}_{\mathrm{w}} "=700 \mathrm{Wcm}^{2}\right)$ .55

26. Fig. 26 Mass transfer rate on surfaces for comparison of impact weight between pillar height and pillar array density $\left(\mathrm{q}_{\mathrm{w}} "=700 \mathrm{~W} / \mathrm{cm}^{2}\right)$ .56

27. Fig. 27 Heat transfer coefficient on surfaces for comparison of impact weight between pillar height and pillar array density $\left(\mathrm{q}_{\mathrm{w}} "=700 \mathrm{~W} / \mathrm{cm}^{2}\right)$ 
28. Fig. 28 Temperature contours on surfaces for comparison of impact weight between pillar height and pillar array density $\left(\mathrm{q}_{\mathrm{w}}{ }^{\prime \prime}=700 \mathrm{~W} / \mathrm{cm}^{2}\right)$ .58

29. Fig. 29 Bottom surface temperature on surfaces with different wettability texture $\left(q_{w}{ }^{\prime \prime}=\right.$ $700 \mathrm{~W} / \mathrm{cm}^{2}$ ) .60

30. Fig. 30 Mass transfer rate on surfaces with different wettability texture $\left(q_{w}{ }^{\prime \prime}=700 \mathrm{~W} / \mathrm{cm}^{2}\right)$

31. Fig. 31 Heat transfer coefficient on surfaces with different wettability texture $\left(\mathrm{q}_{\mathrm{w}}{ }^{\prime \prime}=\right.$ $\left.700 \mathrm{~W} / \mathrm{cm}^{2}\right)$

\section{LIST OF TABLES}

1. Surface topology dimension in comparative studies 


\section{NOMENCLATURE}

$A_{i} \quad$ Volumetric interfacial surface area $\left(\mathrm{m}^{2} / \mathrm{m}^{3}\right)$

$C_{l} \quad$ Mass transfer time relaxation coefficient for liquid phase $(1 / \mathrm{s}) ; C_{l}=1001 / \mathrm{s}$

$C_{v} \quad$ Mass transfer time relaxation coefficient for vapor phase $(1 / \mathrm{s}) ; C_{v}=100 \mathrm{1} / \mathrm{s}$

$D_{\text {sm }} \quad$ Mean Sauter diameter (m)

$E \quad$ Total energy per unit mass $(\mathrm{J} / \mathrm{kg})$

$E_{l} \quad$ Total energy per unit mass of liquid phase $(\mathrm{J} / \mathrm{kg})$

$E_{v} \quad$ Total energy per unit mass of vapor phase $(\mathrm{J} / \mathrm{kg})$

$F \quad$ Volumetric mass flux $\left(\mathrm{kg} / \mathrm{s} \mathrm{m}^{3}\right)$

$F^{\prime} \quad$ Net mass flux over the liquid-vapor interface $\left(\mathrm{kg} / \mathrm{s} \mathrm{m}^{2}\right)$

$F_{\text {vol }} \quad$ Volume force $(\mathrm{N})$

$\vec{g} \quad$ Gravitation acceleration $\left(\mathrm{m} / \mathrm{s}^{2}\right) ;$ standard values $=9.80665 \mathrm{~m} / \mathrm{s}^{2}$

$h_{l v} \quad$ Latent heat between liquid phase and vapor phase $(\mathrm{J} / \mathrm{kg})$

$k \quad$ Thermal conductivity $(\mathrm{W} / \mathrm{m} \mathrm{K})$

$k_{l} \quad$ Thermal conductivity of liquid phase $(\mathrm{W} / \mathrm{m} \mathrm{K})$

$k_{v} \quad$ Thermal conductivity of vapor phase (W/m K)

$k_{B} \quad$ Boltzmann constant $(\mathrm{J} / \mathrm{K})$

M Molecular weight $(\mathrm{kg} / \mathrm{kmole})$

$\dot{m} \quad$ Volumetric mass transfer rate $\left(\mathrm{kg} / \mathrm{m}^{3} \mathrm{~s}\right)$

$\dot{m}_{g \rightarrow l}$ Volumetric mass transfer rate from gas phase to liquid phase (kg/s)

$\dot{m}_{l \rightarrow g}$ Volumetric mass transfer rate from liquid phase to gas phase (kg/s)

$p \quad$ Pressure $(\mathrm{Pa})$ 
$P_{\text {sat }} \quad$ Saturation pressure $(\mathrm{Pa})$

$q_{w-i}^{\prime \prime} \quad$ Net heat flux over the solid-liquid interface

$Q \quad$ Volumetric heat source term $\left(\mathrm{W} / \mathrm{m}^{3}\right)$

$Q_{g \rightarrow l} \quad$ Volumetric heat source term from gas phase to liquid phase $\left(\mathrm{W} / \mathrm{m}^{3}\right)$

$Q_{l \rightarrow g} \quad$ Volumetric heat source term from liquid phase to gas phase $\left(\mathrm{W} / \mathrm{m}^{3}\right)$

$R \quad$ Universal gas constant $(\mathrm{J} / \mathrm{mol} \mathrm{K})$

$r \quad$ Radius (m)

T Temperature $(\mathrm{K})$

$T_{w-i} \quad$ Temperature of solid-liquid interface

$T_{l} \quad$ Temperature of the liquid phase $(\mathrm{K})$

$T_{v} \quad$ Temperature of the vapor phase $(\mathrm{K})$

$T_{\text {sat }} \quad$ Saturation temperature $(\mathrm{K})$

$t \quad$ Time (s)

$V \quad$ Volume $\left(\mathrm{m}^{3}\right)$

$V_{i} \quad$ Volume of species $i\left(\mathrm{~m}^{3}\right)$

$\vec{v} \quad$ Overall velocity vector $(\mathrm{m} / \mathrm{s})$

Greek symbols

$\alpha \quad$ Volume fraction (dimensionless)

$\alpha_{l} \quad$ Volume fraction of liquid phase (dimensionless)

$\alpha_{v} \quad$ Volume fraction of vapor phase (dimensionless)

$\beta \quad$ Accommodation coefficient (dimensionless)

$\theta_{w} \quad$ Contact angle at the wall (degree) 


$\begin{array}{ll}\kappa_{l} & \text { Curvatures of liquid phase }(1 / \mathrm{m}) \\ \kappa_{v} & \text { Curvatures of vapor phase }(1 / \mathrm{m}) \\ \lambda & \text { Thermal conductivity }(\mathrm{W} / \mathrm{m} \mathrm{K}) \\ \lambda_{l} & \text { Thermal conductivity of liquid phase }(\mathrm{W} / \mathrm{m} \mathrm{K}) \\ \lambda_{v} & \text { Thermal conductivity of vapor phase }(\mathrm{W} / \mathrm{m} \mathrm{K}) \\ \mu & \text { Dynamic viscosity (Pa s) } \\ \mu_{l} & \text { Dynamic viscosity of liquid phase }(\mathrm{Pa} \mathrm{s}) \\ \mu_{v} & \text { Dynamic viscosity of vapor phase }(\mathrm{Pa} \mathrm{s}) \\ \rho & \text { Density }\left(\mathrm{kg} / \mathrm{m}^{3}\right) \\ \rho_{i} & \text { Density of species } i\left(\mathrm{~kg} / \mathrm{m}^{3}\right) \\ \rho_{l} & \text { Density of liquid phase }\left(\mathrm{kg} / \mathrm{m}^{3}\right) \\ \rho_{v} & \text { Density of vapor phase }\left(\mathrm{kg} / \mathrm{m}^{3}\right) \\ \sigma & \text { Surface tension }(\mathrm{N} / \mathrm{m})\end{array}$

Abbreviations

CNTs Carbon nanotubes

CSF Continuum surface force

EWOD Electrowetting on dielectric

VOF Volume of Fluid 


\title{
TWO-PHASE FLOW OVER FLOODED MICRO-PILLAR STRUCTURES WITH ENGINEERED WETTABILITY
}

\author{
Zhou Zhou \\ Dr. Chung-Lung Chen, Thesis Supervisor
}

\begin{abstract}
Flooding caused by excessive droplet feeding on heat dissipation areas periodically occurs for droplet-based thermal management. The conventional highly wettable texture of target surfaces, which is designed for thin film evaporation, has negligible effect on improving thermal performance during flooding. This work examines a combination of micro-pillar structures and engineered wettability that aims to improve the liquid-vapor phase change intensity and heat dissipation rate during flooding. Numerical simulation has been made to investigate the thermal and dynamic impact of the proposed combination structure on boiling and evaporation. A transient 3-D volume-of-fluid (VOF) model has been developed to analyze behaviors of bubble growth, coalescence, and departure processes. Parameters including volumetric liquid-vapor mass transfer rate, heat source temperature and heat transfer coefficient are examined. It has been demonstrated that surface wettability gradient in the pillar height direction can effectively facilitate the bubble departure and removal within the pillar forest. Thus smaller bubble size and a lower thermal resistance in the fluid domain can be achieved. The structured surface with higher pillars and denser pillar array is desirable for heat dissipation. The factor of pillar height has more impact on cooling enhancement than pillar array density when the solid-liquid interface area was kept the same. For wettability texture on the micro-pillar structure, the resulting heat transfer performance is determined by a trade-off between the bubble departure improvement within the pillar forest and the bubble pinning at hydrophobic pillar tops.
\end{abstract}




\section{CHAPTER 1: GENERAL INTRODUCTION}

With the inherent requirement of reliable operation, miniaturization, weight reduction and energy efficiency for electronic systems, Moore's Law was continued with smaller size of electronic components and more transistors per unit area. The simultaneous significant increase in power dissipation has led to remarkably challenging thermal issues [1-3]. Improper thermal management will cause continuous temperature increase and eventually lead to electronic failures. High performance chips are expected to generate a heat flux greater than $150 \mathrm{~W} / \mathrm{cm}^{2}$ with localized sub millimeter hot spot heat flux exceeding $1 \mathrm{~kW} / \mathrm{cm}^{2}[4,5]$. The ever increasing integration of density and reliability requirements in the microelectronics industries requires advanced thermal dissipation technology to remove excess heat flux in hotspots with less superheat. Conventional heat transfer from electronic modules to surrounding space has been achieved through the natural, forced, or mixed convection of single phase working fluids. A representative concept by using that mechanism is a microchannel approach, which has been introduced by Tuckerman and Pease three decades ago [6]. Single phase cooling issues were primarily investigated in this conventional system by substantial research efforts [7-16]. However, the cooling efficiency, by using single phase, is directly determined by the sensible heat of the coolant, thus a high volume flow rate for the coolant is required and this approach would face substantial pumping power issues [17, 18], which restrict the cooling effect on higher heat flux conditions, thereby affecting the digital circuits density and performance. Two-phase heat transfer methods, including boiling and thin film evaporation, possess the attractive features with large latent heat transfer by vaporization process. The representative two phase cooling 
method is associated with droplet-based coolant delivery approaches, including electro-wetting cooling, spray cooling etc.

The characteristics of droplet-based coolant delivering thermal management enables precise droplet feeding to the designed area, which can potentially create a more uniform temperature distribution. Furthermore, as opposed to pool boiling or flow boiling systems which always encounter the high incipient superheat problem, droplet-based thermal management like spray cooling can maintain a low superheat, if the thin liquid film is formed at the target surface with appropriate controlled droplet feeding [19, 20]. In addition, droplet-based thermal management can work under higher thermal design limits by providing higher critical heat flux [20].

On the other hand, inadequate coolant feeding frequency by droplet-based thermal management can easily cause periodic flooding. A flooded regime is reported as a periodic scenario occurring in droplet-based thermal management in Hsieh and Shen's work [20, 21]. Due to the favorable superhydrophilic surface for liquid spreading, micro-structures worked efficiently in the thin film and partial dry-out regimes, while for the flooded regime, Hsieh and Yao [20] claimed that negligible effect on heat transfer performance can be induced by highly wettable surface texture. The flooded regime, which occupied about $20 \%$ of the total evaporation time [21], comes with boiling phenomenon which may cause large surface heat flux and surface temperature oscillations. Thus the heat transfer performance in the flooded regime will undoubtedly affect the thermal performance of the entire cooling process. However, boiling and evaporation phenomena during flooding were rarely mentioned in former studies.

In this paper, our research is to numerically investigate the boiling and evaporation process during flooding. A micro-pillar structure combined with engineered wettability gradient 
is proposed and modeled for understanding its capability on cooling enhancement and facilitating bubble departure. The impacts of various micro-pillar dimensions as well as wettability texture on two-phase flow dynamics and heat transfer characteristics during flooding were examined by several comparative studies. The transient three-dimensional VOF models have been used, which have the capability to track the liquid-vapor interface and bubble trajectories continuously for visualizing multi-bubble dynamics. The effect of surface wettability was modeled by specifying the wall adhesion angle in conjunction with the surface tension model. The solid material and working fluid are aluminum and water respectively. Evaporation, boiling and condensation at the liquid-vapor interface are modeled by using the Hertz-Knudsen equation derived from kinetic theory. Control variables for comparative studies include pillar height, pillar array density and wettability texture on pillar top surface. 


\section{CHAPTER 2: LITERATURE REVIEW}

Three sections are included in this chapter which provides a literature review of past research in the field of two-phase flow about droplet-based thermal management, bubble dynamics and phase change investigation with VOF method.

\subsection{Droplet-based Thermal Management}

\subsubsection{Electrowetting Thermal Management}

As a paradigm-shift technique, electrowetting on dielectric (EWOD) uses electric potential to manipulate the movement of a liquid droplet on a dielectric surface. Droplets are actuated independently in user-defined patterns over an array of electrode, eliminating the demand for an external pump. In this way, droplets can be precisely delivered to the hot spot.

Cheng and Chen [22] developed an analytical resistance-capacitor network model for estimating the EWOD driving force under AC. They accomplished 2D transport of a liquid droplet in any desired flow path with only 40 volts. They further demonstrated the adaptive and active thermal management of on-chip hot spot using EWOD. The hot spot surface was successfully cooled down by more than $40^{\circ} \mathrm{C}$ within 2 s. They pointed out the critical development issues of EWOD, which include the fast droplet transport for high heat-flux situations, real-time hot spot detection via IR imaging, and dynamic cooling target tracking protocol.

In another work published by Cheng and Chen [23], a hot spot with $27 \mathrm{~W} / \mathrm{cm}^{2}$ was reported to be rapidly cooled down by $30^{\circ} \mathrm{C}$ by a $14 \mu \mathrm{L}$ droplet, which was transmitted by an 
actuation mechanism. They also incorporated carbon nanotubes (CNTs) in a parallel-plate EWOD configuration. They indicated that the multiscale roughness which is optimized in favor of the Cassie state can lead to superhydrophobicity and reduce the pressure loss significantly.

Paik et al. [24] manipulated the discrete liquid droplet immersed in oil. They demonstrated that the minimum voltage required to oscillate a microliter-sized droplet decreased as much as $30 \%$ for a $50 \mathrm{~K}$ temperature increase under a fixed frequency. Temperature related parameters including interfacial tension and oil viscosities are investigated. The increased flow at higher temperatures makes this platform ideal for active chip cooling applications.

Mohseni [25] proposed active heat management by actuating the discrete droplet of metals/alloys. He indicated that by using liquid metal or alloys at room temperature the heat transfer of a coolant was enhanced by more than two orders of magnitude. Another idea introduced by him is that the EWOD is an efficient, low power consumption actuation method for pumping liquids at micro-scales. Liquid velocity above $10 \mathrm{~m} / \mathrm{s}$ can be observed with extremely low pumping power consumption and at low actuation voltage of about $2 \mathrm{~V}$.

Mohseni and Baird [26] tested the digitized heat transfer performance using liquid alloy galinstan, which is nontoxic and inexpensive. Significant improvement was achieved due to galinstan's orders of magnitude higher values of thermal mass and thermal conductivity. They also derived the nature of EWOD force and the velocity of EWOD actuated droplets.

Bahadur and Garimella [27] developed a model that predicts transient electrowettinginduced droplet motion by analyzing droplet motion between two flat plates under the influence of an electrowetting voltage. They also analyzed the influence of interfacial energies, surface roughness parameters and electric fields in determining the apparent contact angle of a droplet in the Cassie and Wenzel states under the influence of an electrowetting voltage. An energy 
minimization-based modeling approach is used to establish preliminary criteria to design rough surfaces for use in the hot-spot cooling application. The concept of an electrically tunable thermal resistance switch for hot-spot cooling applications is introduced and analyzed.

Kumari and Garimella [28] quantified the heat dissipation capacity of actuated droplets through detailed modeling and experimental efforts. The modeling involves three-dimensional transient numerical simulations of a droplet moving under the action of gravity or electrowetting on a single heated plate and between two parallel plates. They observed the fluid circulation pattern, which shows a strong influence on thermal transport and heat transfer coefficient. The complexity of the transient flow patterns emphasizes the necessity for analysis of the flow field and its effect on the heat removal capacity of droplet-based systems. The heat dissipation capacity of electrowetting-based systems is seen to be comparable to microchannel cooling systems operating at the same pumping power. The advantage of an electrowetting-based system, including enhanced reconfigurable flow control, noiselessness without mechanical moving parts and ease of integration with existing framework for microelectronics packaging, was reported.

The volume of fluid (VOF) method was used by Annapragada et al. [29] to predict the transient behavior of water droplets on smooth hydrophobic surfaces under electrowetting actuation. Electrowetting and dynamic frictional forces are included as an effective dynamic contact angle through a force balance at the contact line. The predictions are in agreement with experimental measurements. The internal fluid motion is explained, and the droplet motion is shown to initiate from the contact line. They also developed an approximate mathematical model to understand the physics of the droplet motion and to describe the overall droplet motion and the contact line velocities. 
The theoretical and numerical results for digitized heat transfer investigated by Baird et al. [30] reflect the enhancement of the heat transfer coefficient brought by droplet internal circulation, and summarized that the digitized heat transfer has the capability for active, ondemand suppression of transient hot spots.

\subsubsection{Spray Cooling Thermal Management}

Spray cooling denotes the cooling process when liquid forced through a small orifice shatters into a dispersion of fine droplets which then impact a heated surface. The droplets spread on the surface and evaporate or form a thin liquid film, removing large amounts of energy at low temperatures due to the latent heat of evaporation in addition to single-phase convection effects. Higher heat transfer rates, the possibility of uniformly cooling large surfaces, low droplet impact velocity, and no temperature overshoot are the advantages of spray cooling [31].

Kim [31] gives a review for spray cooling heat transfer in aspects of the spray cooling heat transfer mechanism and some models, effects of non-condensable gas, surface enhancement, spray inclination and gravity were also contained in the discussion. He concluded from the majority of the experimental data in the review that spray cooling mechanisms in both singlephase and two-phase regimes have yet to be identified. In his point of view, new experimental techniques to measure spray parameters and to record high speed videos are required for further understanding of spray cooling.

Yang et al. [32] discussed the effect of liquid film thickness on heat transfer. In spray cooling, if the number of droplets falling on the surface is large enough, a uniform continuous film on the surface is then formed. The liquid film and its thickness were reported to play an 
important role in the nucleate boiling heat transfer contribution of spray cooling. Depending on the heat flux, the heat transfer coefficient can increase by as much as 50 percent as the liquid level is reduced, until dryout occurs.

Horacek et al. [33] measured wall heat transfer during spray cooling. The contact line length was shown to correlate with wall heat flux rather than the surface wetted area. The presence of non-condensable gas was verified to cause a shift in the saturation temperature of the liquid and increased the subcooling of the liquid being sprayed onto the surface.

Spray cooling on microstructured surfaces, which include microscale indentations and surface protrusions, was studied by $\mathrm{H}$. Bostanci et al [34]. Heat flux up to $500 \mathrm{~W} / \mathrm{cm} 2$ was removed. For microstructured surfaces with protrusions and indentations, the increases in the heat transfer coefficient were $112 \%$ and $49 \%$ over that of a smooth surface, respectively.

Another spray cooling investigation using ammonia by Bostanci et al. [35] shows a high CHF value of approximately $1100 \mathrm{~W} / \mathrm{cm}^{2}$. The heat transfer enhancement of microstructure surfaces was validated again over the reference smooth surface. They demonstrated that enhanced surfaces are capable of retaining more liquid compared to a smooth surface, and efficiently spread the liquid film via capillary force within the structures. Fluid retention delays the occurrence of dry patches at high heat fluxes, and leads to higher CHF.

Zhang el al. [36] experimentally studied the spray cooling heat transfer on flat and microstructure enhanced surfaces. In both single phase and two phase regions, the heat transfer enhancement for structured surfaces was observed and compared with the enhanced surface. The structure with smaller feature size had better heat transfer performance. The optimal flow rate for the enhanced surface was found to be larger than that of the flat surface, while the optimal orifice-to-surface distance for the enhanced surface is smaller than the flat one. The optimal 
distance decreases as the groove size is reduced. The effect of inclination angle and surface roughness were discussed too.

\subsection{Bubble Dynamics}

$\mathrm{Li}$ and Peterson [37] evaluated the boiling incipience and bubble dynamics on a microfabricated heater using a high-speed digital camera. It is reported that explosive boiling can take place on a smooth surface, with roughness equal to the critical bubble size, no matter how slow the heating rate. In addition, the boiling incipience and bubble behavior from a smooth surface were observed to be much different than for the boiling taking place on a rough surface, even if the size of the nucleation cavity is in the range of microns, which represents the more conventional boiling phenomena, and can be explained by Hsu's proposed theory of bubble survival. Bubble growth from a smooth surface was found to have a dynamic stage. The duration of the dynamic stage depends on heating rate and pulse duration, the presence of noncondensable gas, and the degree of subcooling present.

Luke and Cheng [38] Carried out the experiments on bubble formation and heat transfer measurements. The number and location of active nucleation sites was obtained in images of the video sequence and some selected results for bubble departure diameter and frequency. Results for the bubble formation on a horizontal copper tube in boiling propane were discussed. Additionally, the fluctuation by time and in the location on the tube is recorded. Individual bubble growth on its nucleation site and movement along the heated tube was tracked. Their results showed that the bubbles sliding up the superheated tube surface grow at a similar rate as those staying on their nucleation site. 
Frank et al. [39] investigated the nucleation and growth of $\mathrm{CO}_{2}$ bubbles. At an immobile nucleation site the bubble grows rapidly followed by a linear increase in bubble diameter with time. After reaching a critical size, the bubble detached from its stagnant point and rose in the liquids with an exponential temporary increase in both the diameter and distance. The growth rate and flow fields around a $\mathrm{CO}_{2}$ micro-bubble were measured. Relevant information including fluid hydrodynamics, mass transfer and interfacial phenomena in the microscale quantified the dynamic bubble growth at a nucleation site and corresponding flow fields around the bubble.

Chao et al. [40] studied the formation and growth processes of a bubble in the vicinity of graphite micro-fiber tips on metal-graphite composite boiling surfaces and their effects on boiling behavior. It was discovered that a large number of micro bubbles are formed first at the micro scratches and cavities on the metal matrix in pool boiling. Due to the hydrophobic feature of graphite, once the growing micro bubbles touched the graphite tips, those bubbles were sucked by the tips and merged together into larger micro bubbles sitting on the end of the tips. The micro bubbles coalesced to form macro bubbles, each spanning several tips. The necking process of a detaching macro bubble was also analyzed. They revealed that a liquid jet is produced by a sudden break-off of the bubble throat. The composite surfaces were validated to not only have higher temperatures in micro- and macro-layers but to also raise the frequency of the bubble departure, which increased the average heat fluxes in both the bubble growth stage and in the bubble departure period.

Siedel et al. [41] described experimental results of pentane pool boiling on either a single nucleation site or on two adjacent nucleation sites. Bubbles growth was recorded and oscillations were observed during growth, showing the interaction of one bubble with the preceding bubble released from the same nucleation site, which illustrated that the bubble interaction is therefore a 
significant factor that should definitely be taken into account while modeling of bubble growth. The bubble volume at detachment is independent of the wall superheat, whereas the growth time is dependent on the superheat. The bubble growth law they summarized is very different from most analytical models, especially on the issue of vapor production rate during the bubble growth. Bubble coalescence between two neighboring bubbles had also been studied, which showed that coalescence does not have a great impact on vapor production during merging.

\subsection{Phase Change Investigation with VOF Method}

A numerical model was developed by Ranjan et al. [42] for the evaporating liquid meniscus in different wick microstructures under saturated vapor conditions. Steady evaporation was modeled and the interface shape was assumed to be static and obtained by Surface Evolver during evaporation. Evaporation at the interface was modeled by using heat and mass transfer rates obtained from kinetic theory. The evaporation heat transfer was noted to occur from the thin-film region and occupied more than $80 \%$ of the liquid meniscus. Evaporation heat transfer decreases with increasing contact angle and with increasing wick character length, because of a smaller thin-film area.

The VOF model was widely used in describing the evaporation and boiling phenomena together with appropriately implemented source terms. Yang et al. [43] conducted the boiling flow experiment of R141b in a horizontal tube. A corresponding numerical simulation was carried out using the VOF multiphase model. Good consistence of phase evolution was achieved between the numerical predictions and experimental observations. The simulation result was 
reported to exhibit the evolution of flow mode comprehensively, depending on the thermal and hydro-dynamical conditions.

A three dimensional VOF simulation of R134a condensation process has been reported by Riva and Col [44]. Computational results displaying the evolution of the vapor-liquid interface and heat transfer coefficient are reported and validated against experimental data.

Zhang et al. [45] numerically investigated the forced convective condensation in miniature channels. They used the VOF method to simulate the capillary blocking phenomenon, which happens due to condensation in a horizontal miniature tube and between parallel plates. Nusselt condensation of steam vapor on a vertical flat plate as well as forced film condensation on a horizontal flat plate was numerically solved for comparison with the analytical solution. The overall verification result showed agreement between simulation and the analytical solution.

The refrigerant flow boiling in a horizontal serpentine tube experiment and numerical study with VOF model was conducted by Wu et al. [46]. The comparison indicates that both the experimental observation and numerical simulation are consistent with each other. Particularly, the simulation well explains the effects of phase redistribution and thermal non-equilibrium release observed in the experiments.

De Schepper et al. [47] performed 3D-simulations of the evaporation process of a hydrocarbon feedstock. The heating, evaporation and boiling phenomena occurring were modeled by VOF model with a source term that had been derived from the Hertz-Knudsen equation. The simulation results show a succession of horizontal two-phase flow regimes in agreement with the literature.

Fang et al. [48] present a 3D numerical simulation result of the vapor-venting process in a rectangular microchannel with a hydrophobic porous membrane for phase-separation. The 
simulation is based on the VOF method together with capillary force models and an interphase mass transfer model which is derived from the Hertz-Knudsen equation. A comparison between the vapor-venting channel and the conventional channel for the two-phase flow patterns, the pressure drop, and the temperature profile was discussed for providing insight into the optimization of vapor-venting heat exchangers. 


\section{CHAPTER 3: NUMERICAL MODEL}

\subsection{Volume of Fluid Method}

The Volume of Fluid Model (VOF) model is a surface tracking approach used for two or more immiscible fluids where the evolution and location of the interface between the fluids is of interest. This technique is applied to updating the phase volume fraction in the fixed grid. In each computational cell the fraction of each fluid is tracked. Thus the transient tracking of the liquidgas interface can be achieved by using the VOF model in this research, for visualizing the bubble dynamic motion, bubble merging, and bubble departure process. The impacts related to the bubble dynamics on two-phase cooling performance can also be understood by solving the VOF model as well as the continuity equation, momentum equation, energy equation and mass transfer equation.

\subsubsection{Phase Conservation}

In the VOF model, a scalar field representing the portion of phase volume occupation in a certain computational cell is defined as phase volume fractions. In each control volume, the sum of liquid and gas phase volume fractions should keep a constant,

$$
\alpha_{l}+\alpha_{v}=1
$$

It is assumed that the cell represents the liquid region if $\alpha_{v}=0$, the cell represents the gas region if $\alpha_{v}=1$, and $0<\alpha_{v}<1$ indicates the cell contains both liquid phase and vapor phase, and the liquid-vapor interface is located inside the cell. 


\subsubsection{Physical Properties}

On condition that the volume fraction of each phase is known at each position, the physical properties in any computational cell are either purely representative of liquid phase or gas phase, or representative of a mixture of the phases. Based on the local value of the volume fraction $\alpha$, the properties for each cell can be obtained by following equations.

$$
\begin{gathered}
\rho=\alpha_{l} \rho_{l}+\alpha_{v} \rho_{v} \\
\mu=\alpha_{l} \mu_{l}+\alpha_{v} \mu_{v} \\
\lambda=\alpha_{l} \lambda_{l}+\alpha_{v} \lambda_{v} \\
E=\left(\alpha_{l} \rho_{l} E_{l}+\alpha_{v} \rho_{v} E_{v}\right) /\left(\alpha_{l} \rho_{l}+\alpha_{v} \rho_{v}\right)
\end{gathered}
$$

\subsubsection{Interface Representation}

In the VOF model, the phase volume fraction field is updated according to the velocity field and the phase volume fraction field in the previous time step. Based on the volume fraction of each phase in that cell and in the neighboring cells, the interface shape of each cell is approximated using piecewise-linear approach by the geometric reconstruction scheme, which is the most accurate scheme in software ANSYS FLUENT. The interface between the two fluids is assumed to have a linear slope within each cell. Therefore the computational cells containing the interface are cut by a line segment, such that the phase volumes in the cell correspond with the values of the phase volume fraction in that cell. The resulting interface polygon is then used for calculation of the advection of fluid through the cell faces. 
In geometric reconstruction scheme, the location of the linear interface of each partially filled cell is solved in the first step, based on information of the volume fraction and its derivatives in the cell. Then the advecting amount of fluid through each cell face is calculated using the linear interface representation and velocity distribution information on the face. The third step is volume fraction calculation in each cell, based on the balance of fluxes calculated during the previous step.

\subsection{Governing Equations}

With the concept of volume fraction, the VOF model can model two fluids by solving a single set of equations including the continuity equation, momentum equation and energy equation. Tracking the interface between liquid phase and gas phase in the system is accomplished by solving the continuity equations with mass transfer source term as follow:

$$
\begin{aligned}
& \frac{\partial \alpha_{l}}{\partial t}+\nabla \cdot\left(\vec{v} \alpha_{l}\right)=\frac{\dot{m}_{g \rightarrow l}}{\rho_{l}} \\
& \frac{\partial \alpha_{v}}{\partial t}+\nabla \cdot\left(\vec{v} \alpha_{v}\right)=\frac{\dot{m}_{l \rightarrow g}}{\rho_{v}}
\end{aligned}
$$

where $\dot{m}_{g \rightarrow l}$ and $\dot{m}_{l \rightarrow g}$ is the source term indicating the volumetric mass transfer rate through the bubbles interface. The momentum equation, which is shared between phases through the physical properties $\mu$ and $\rho$, is shown below:

$$
\frac{\partial}{\partial t}(\rho \vec{v})+\nabla \cdot(\rho \vec{v} \vec{v})=-\nabla p+\nabla \cdot\left[\mu\left(\nabla \vec{v}+\nabla \vec{v}^{T}\right)-\frac{2}{3} \mu \nabla \cdot \vec{v} I\right]+\rho \vec{g}+\vec{F}_{v o l}
$$

The gravity and the volumetric surface tension force $\vec{F}_{v o l}$ were taken into account, which will be discussed in section 3.3. The energy equation shared between phases is: 


$$
\frac{\partial}{\partial t}(\rho E)+\nabla \cdot(\vec{v}(\rho E+p))=\nabla \cdot(k \nabla T)+Q
$$

The energy, $E$, and temperature, $T$, are treated as mass-averaged variables in VOF model. The properties $\rho$ and $k$ are shared by the phases. The energy source term can be represented as follow:

$$
\begin{aligned}
& Q_{l \rightarrow g}=\dot{m}_{g \rightarrow l} h_{l v} \\
& Q_{g \rightarrow l}=\dot{m}_{l \rightarrow g} h_{l v}
\end{aligned}
$$

\subsection{Surface Tension}

Surface tension is a force; the result of attractive forces between molecules in a fluid. Because of the existence of higher pressure on the concave side of the spherical interface, the surface tension force at interface acts to maintain the equilibrium between the radially outward pressure gradient force across the interface and the radially inward intermolecular attractive force. When one of two separated fluids is not in the form of spherical bubbles, the surface tension acts to minimize the surface free energy by decreasing the area of the interface. The following Young-Laplace equation relates the pressure difference across the interface to the interfacial tension and the geometry of the interface at equilibrium:

$$
P_{\mathrm{I}}-P_{\mathrm{II}}=\sigma\left(\frac{1}{r_{1}}+\frac{1}{r_{2}}\right)
$$

where $r_{1}$ and $r_{2}$ represent the two radii in orthogonal directions. The pressure inside the drop or bubble $P_{\mathrm{I}}$ exceeds the pressure outside $P_{\mathrm{II}}$.

For the cells containing the vapor-liquid interface, the surface tension is computed by the continuum surface force (CSF) model proposed by Brackbill [49] in ANSYS FLUENT. The surface tension can be written in terms of the pressure jump across the surface with finite 
thickness. By using the divergence theorem, the force at the surface can be expressed as a volume force and act as a source term in the momentum equation with the following form:

$$
F_{v o l}=\sigma \frac{\alpha_{l} \rho_{l} \kappa_{v} \nabla \alpha_{v}+\alpha_{v} \rho_{v} \kappa_{l} \nabla \alpha_{l}}{\frac{1}{2}\left(\rho_{l}+\rho_{v}\right)}
$$

where $\sigma$ is the interfacial tension force between the liquid and vapor, and was specified as $0.0589 \mathrm{~N} / \mathrm{m}$ in this study. In CSF mode the surface curvature is computed from local gradients in the surface normal at the interface. With the surface normal $n$ defined as the gradient of the volume fraction $\alpha$,

$$
n=\nabla \alpha
$$

the curvature is defined in terms of divergence of the unit normal, $\hat{n}$ [49]:

$$
\kappa=\nabla \cdot \hat{n}
$$

where

$$
\hat{n}=\frac{n}{|n|}
$$

The curvatures of the liquid and vapor phases are thereby defined as

$$
\begin{aligned}
& \kappa_{l}=\nabla \cdot \frac{\nabla \alpha_{l}}{\left|\nabla \alpha_{l}\right|} \\
& \kappa_{v}=\nabla \cdot \frac{\nabla \alpha_{v}}{\left|\nabla \alpha_{v}\right|}
\end{aligned}
$$

\subsection{Wall Adhesion}

In VOF model, with the wall adhesion angle in conjunction with the surface tension model, the contact angle that the fluid is expected to contact with the wall is applied to adjust the 
surface normal in cells near the wall. This dynamic boundary condition enables the adjustment of the interface curvature near the wall. If $\theta_{w}$ is defined as the contact angle at the wall, the surface normal at the cell next to the wall is

$$
\hat{n}=\hat{n}_{w} \cos \theta_{w}+\hat{t}_{w} \sin \theta_{w}
$$

where $\hat{n}_{w}$ and $\hat{t}_{w}$ represent the normal unit vectors and tangential unit vectors to the wall, respectively. The local curvature of the surface is determined by the combination of this contact angle with the surface normal one cell away from the wall. The body force term is adjusted by this local curvature in the surface tension calculation.

\subsection{Mass Transfer Model}

The liquid-vapor mass transfer rate for evaporation and boiling processes is a critical issue for two-phase flow problems. Based on the temperature field, the interface mass transfer can be calculated, and represented by the source term in the continuity equation and energy equation. Different forms of the phase change rate were used by researchers. The energy source term with the assumption of linear temperature distribution in the liquid layer is formulated by Nusselt [50] when solving the laminar film condensation problem, which is can be introduced by:

$$
Q_{g \rightarrow l}=k_{l} \frac{T_{s a t}-T_{w}}{\delta_{l}} \frac{A}{V}
$$

where $A$ is the area of the interface and $V$ is the cell volume. Wang and Sunden [51] simulate the phase change phenomena based on the temperature gradient at the interface, they directly 
calculate the normal component of the heat transfer rate vector to the liquid-vapor interface as follows:

$$
Q_{l \rightarrow g}=k_{l} \frac{\partial T}{\partial|\vec{n}|} \frac{A}{V}
$$

where $\partial T / \partial|\vec{n}|$ is the temperature gradient at the interface. In NEPTUNE CFD theory manual the energy source term is dependent on the ratio between temperature difference and enthalpy difference as follows:

$$
Q_{g \rightarrow l}=\frac{H T C_{L}\left(T_{s a t}-T_{l}\right)}{h_{v, s a t}-h_{l}} h_{l v}
$$

where $H T C_{L}$ represents the liquid heat transfer coefficient; $h_{v, \text { sat }}$ and $h_{l}$ stand for the saturation enthalpy for the vapor and liquid enthalpy, respectively. In the study of Aghanajafi and Hesampour [52], the source term in the energy and VOF equation is determined using an energy balance at the interface. It can be expressed as:

$$
Q=\left[\alpha_{l} k_{l} \nabla T_{l}-\alpha_{v} k_{v} \nabla T_{v}\right] \nabla \alpha_{l}
$$

However, all the introduced mass transfer models above have corresponding limitations. The linear temperature distribution was assumed at Nusselt's model, which is not able to describe a complex temperature field precisely. Wang and Aghanajafi's model requires an existing interface for mass transfer. Otherwise, the term $\frac{\partial T}{\partial|\vec{n}|}$ and $\nabla \alpha_{l}$ will be zero, which means new bubbles cannot be generated based on this model. For Lavieville's model, the volume fraction term is not included, therefore the bubble dynamic tracking cannot be achieved.

In the two-phase system with boiling, evaporation, and condensation, the most commonly used model is based on the well-known Hertz-Knudsen formula [53], which is derived from 
kinetic theory for flat interfaces. It is used for the calculation of the net mass flux over the vapor/gas-liquid interface as follows:

$$
F^{\prime}=\beta \sqrt{\frac{M}{2 \pi R}}\left(\frac{P^{*}}{\sqrt{T_{v}}}-\frac{P_{s a t}}{\sqrt{T_{l}}}\right)
$$

where $\mathrm{P}^{*}$ represents the partial vapor pressure at the interface on the gas side. In the case of evaporation, the accommodation coefficient $\beta$ is defined as the portion of liquid molecules going to the vapor/gas surface and becoming absorbed by this surface. By approximation with Clausius-Clapeyron equation

$$
\frac{d P}{d T}=\frac{h_{l v}}{T\left(v_{g}-v_{l}\right)}
$$

and considering the volumetric interfacial surface area with the relation of interfacial area density

$$
\frac{A_{i}}{V_{\text {cell }}}=\frac{6}{D_{s m}} \alpha
$$

where $V_{c e l l}$ is the cell volume and $D_{S m}$ represents the mean Sauter diameter [54]. The mass flux over the liquid vapor interface can be represented with

$$
F=F^{\prime} \frac{A_{i}}{V_{c e l l}}=\frac{6}{D_{S m}} \alpha \beta \sqrt{\frac{M}{2 \pi R T_{s a t}}}\left(\frac{h_{l v}}{1 / \rho_{v}-1 / \rho_{l}}\right) \frac{T-T_{s a t}}{T_{s a t}}
$$

Mass transport in this simulation was implemented using the evaporation-condensation mechanistic model [55] in FLUENT. This model primarily concerns phase change at a quasi thermo-equilibrium state, and depends on the temperature difference between local temperature and saturation temperature. If local temperature is higher than the saturation temperature, the evaporation and boiling occurs, liquid water mass is transferred to a water vapor species of gas 
phase. Otherwise condensation will take place. The direction and magnitudes of mass transfer can be described based on different temperature regimes as follows

$$
\begin{gathered}
\dot{m}_{l \rightarrow g}=\left\{\begin{array}{cl}
C_{l} \cdot \alpha_{l} \rho_{l}\left(T_{l}-T_{\text {sat }}\right) / T_{\text {sat }} & T_{l}>T_{\text {sat }} \\
0 & T_{l} \leq T_{\text {sat }}
\end{array}\right. \\
\dot{m}_{g \rightarrow l}=\left\{\begin{array}{cl}
C_{v} \cdot \alpha_{v} \rho_{v}\left(T_{v}-T_{\text {sat }}\right) / T_{\text {sat }} & T_{v}>T_{\text {sat }} \\
0 & T_{v} \leq T_{\text {sat }}
\end{array}\right.
\end{gathered}
$$

$\dot{m}_{l \rightarrow g}$ represents the rate of mass transfer from the liquid phase to vapor phase, and has units of $\mathrm{kg} /\left(\mathrm{s} \cdot \mathrm{m}^{3}\right)$, which is regarded as the source term in the continuity equation and energy equation. $\alpha$ and $\rho$ are the phase volume fraction and density, respectively. Thus the source term in the energy equation can be obtained by the multiplying of the mass transfer rate by the latent heat. $C_{l}$ and $C_{v}$ are coefficients and can be interpreted as relaxation time.

Based on Eq. (2), the introduced coefficients $C_{l}$ and $C_{v}$ can be represented by

$$
\begin{gathered}
C_{l}=\frac{6}{D_{S m}} \sqrt{\frac{\mathrm{M}}{2 \pi \mathrm{RT}_{s a t}}} \frac{\rho_{v} h_{l v}}{\rho_{l}-\rho_{v}} \\
C_{v}=\frac{6}{D_{S m}} \sqrt{\frac{\mathrm{M}}{2 \pi \mathrm{RT}}{ }_{s a t}} \frac{\rho_{l} h_{l v}}{\rho_{l}-\rho_{v}}
\end{gathered}
$$

Both $C_{l}$ and $C_{v}$ were specified as $100 \mathrm{~s}^{-1}$ for this study. The same value has been used in studies proposed by Yang et al [43], Fang et al [48], and Wei et al[56]. The $100 \mathrm{~s}^{-1}$ validated by Yang et al [43] and the numerical results was in good agreement with experimental observations. 


\subsection{Computational Domain}

\subsubsection{Definition of Flooding Scenario and Boundary Settings}

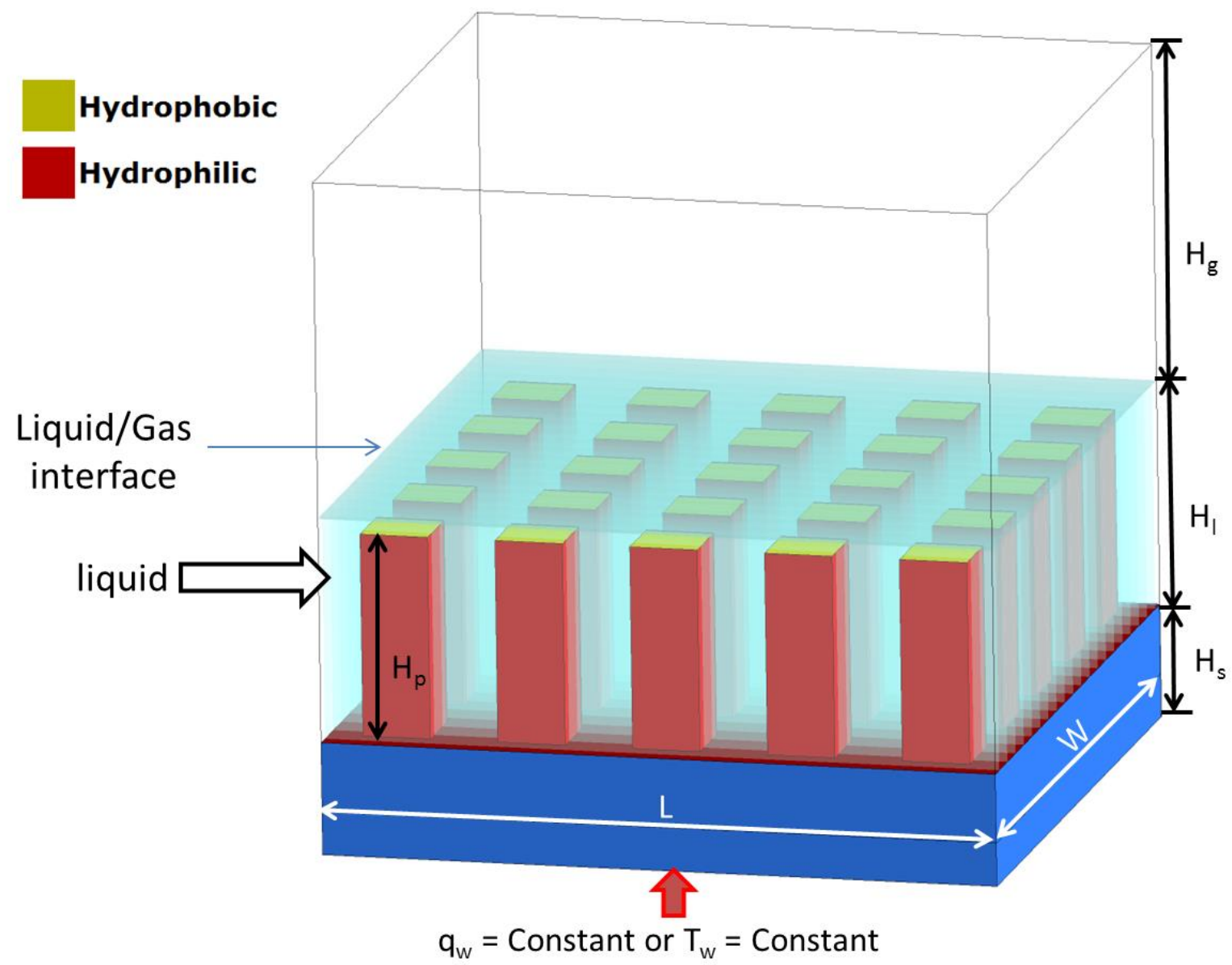

Fig. 1 Computational domain

Fig. 1 exhibits the computational domain during a flooding scenario, which is composed of solid, liquid and gas parts. $\mathrm{L}$ and $\mathrm{W}$ represent the length and width of the domain. The height of solid, height of liquid, and height of gas are denoted by $\mathrm{H}_{\mathrm{s}}, \mathrm{H}_{1}$ and $\mathrm{H}_{\mathrm{g}}$ respectively. The height of the domain, $\mathrm{H}$, is the sum of the height of solid, liquid and gas. Pillar height was symbolized with $\mathrm{H}_{\mathrm{p}}$. The flooding situation is defined as the pillar being fully immersed by the liquid film, 
that is $\mathrm{H}_{1}>\mathrm{H}_{\mathrm{p}}$. The four vertical boundary surfaces in this 3-D domain are considered to be periodic boundaries, which enables the limited computational domain to be considered as a horizontal extensive domain. The energy was input from the solid bottom surface and left from the defined outlet on the top. The outlet of the computational domain, located on the top of the computational domain, was defined as a pressure outlet to allow free access for gas phase mixture containing both species of water vapor and air. The backflow gas species was defined as dry air with temperature equal to $370 \mathrm{~K}$. Based on the hydrophilic solid substrate, surface wettability modification is made upon the pillar's top surface to become hydrophobic.

\subsubsection{Microstructure topologies}

Table 1 summarizes the models of different structures built to investigate the cooling effect brought by the structure's dimensions and arrangement. A total of eight surfaces, including five micro-structured surfaces and three kinds of flat surfaces, have been modeled and simulated for understanding the influence of microstructure geometry on thermal performance as well as surface wettability patterns on bubble dynamic behavior.

\begin{tabular}{|c|c|c|c|c|c|c|c|c|}
\hline Symbol & H0A33_25 & H3A33_25 & H5A33_25 & H7A33_25 & HOA55_9 & H5A55_9 & Hydrophilic & $\begin{array}{l}\text { Hydrophilic_ } \\
\text { H5A33_25 }\end{array}$ \\
\hline flat/structured surface & flat & structure & structure & structure & flat & structure & flat & structure \\
\hline $\begin{array}{l}\text { Pillar array density (number of hydrophobic } \\
\text { spots in domain) }\end{array}$ & $25(5 \times 5)$ & $25(5 \times 5)$ & $25(5 \times 5)$ & $25(5 \times 5)$ & $9(3 \times 3)$ & $9(3 \times 3)$ & 0 & $25(5 \times 5)$ \\
\hline Cross-Section area, L by $\mathrm{W}(\mu \mathrm{m}$ by $\mu \mathrm{m})$ & 3 by 3 & 3 by 3 & 3 by 3 & 3 by 3 & 5 by 5 & 5 by 5 & None & 3 by 3 \\
\hline Height of Pillars, $H_{p}(\mu m)$ & 0 & 3 & 5 & 7 & 0 & 5 & 0 & 5 \\
\hline contact angle of hydrophobic surface & $108^{\circ}$ & $108^{\circ}$ & $108^{\circ}$ & $108^{\circ}$ & $108^{\circ}$ & $108^{\circ}$ & None & None \\
\hline contact angle of hydrophilic substrate & $5^{\circ}$ & $5^{\circ}$ & $5^{\circ}$ & $5^{\circ}$ & $5^{\circ}$ & $5^{\circ}$ & $5^{\circ}$ & $5^{\circ}$ \\
\hline
\end{tabular}

Table 1 Surface topology dimensions in comparative studies 
For all cases the dimensions of the computational domain are $\mathrm{H}=30 \mu \mathrm{m}, \mathrm{L}=30 \mu \mathrm{m}$ and $\mathrm{W}=30 \mu \mathrm{m}$. The thickness of the solid substrate $\mathrm{H}_{\mathrm{s}}=5 \mu \mathrm{m}$. All micro-pillars are immersed by a water film with a thickness $\mathrm{H}_{1}=10 \mu \mathrm{m}$. The remaining space is occupied by vapor/air mixture, with height $\mathrm{H}_{\mathrm{g}}=15 \mu \mathrm{m}$. For the solid substrate, two thermal boundary conditions were imposed on the bottom surface: a constant temperature boundary condition with $\mathrm{T}_{\mathrm{w}}=400 \mathrm{~K}$, and a constant heat flux boundary condition with $700 \mathrm{~W} / \mathrm{cm}^{2}$. The corresponding initial temperature for the whole domain was set to $370 \mathrm{~K}$ for both cases. The solid material is set as aluminum. The variation range for pillar height is $3 \mu \mathrm{m}$ to $7 \mu \mathrm{m}$, which is lower than the thickness of the liquid film of $10 \mu \mathrm{m}$, thus ensuring the flooded condition. The pillar cross-sectional area ranges from $\mathrm{L}=3 \mu \mathrm{m}$ by $\mathrm{W}=3 \mu \mathrm{m}$ to $\mathrm{L}=5 \mu \mathrm{m}$ by $\mathrm{W}=5 \mu \mathrm{m}$. The contact angle for the hydrophilic substrate is $5^{\circ}$, and for the hydrophobic surface pattern is $108^{\circ}$.

Fig. 2 displays the corresponding configurations of micro-structured surfaces and flat surfaces that were listed in Table 1.

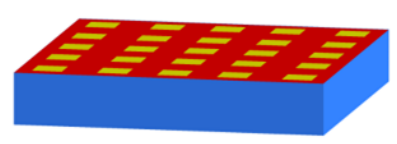

H0A33_25

Hydrophobic

Hydrophilic

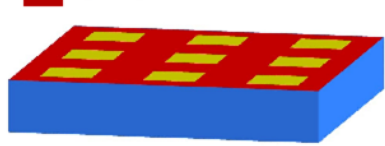

H0A55_9



H3A33_25

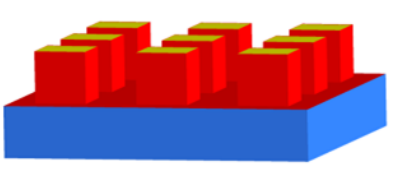

H5A55_9

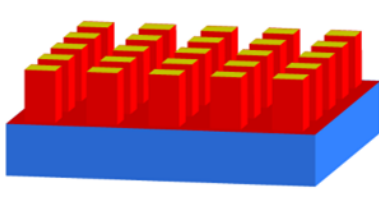

H5A33_25



Hydrophilic

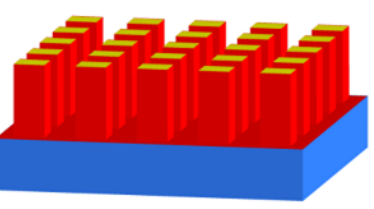

H7A33_25

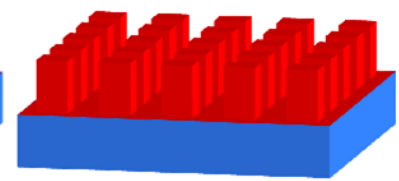

Hydrophilic_H5A33_25

Fig. 2 Scheme for structure geometry 
The flat surface in Table 1 includes a purely hydrophilic surface (Hydrophilic) and a hydrophilic surface with a hydrophobic spot pattern (H0A33_25 and H0A55_9). The structured surfaces are composed of several arrays of pillars. Besides the purely hydrophilic structured surface (Hydrophilic_H5A33_25), the other structured surfaces are set with hydrophilic substrate and relatively hydrophobic pillar-top surfaces, which are designed for pulling bubbles up and facilitating bubble departure. The control variables include micro-pillar height, pillar array density, and wettability texture on the microstructure. The pillar array density is defined as pillar number included per unit area within the computational domain when the solid and liquid volumes are kept the same. Four comparative studies have been conducted by using the structures in Table 1. The objectives are summarized as follows:

First, to identify the micro-pillar height's effect of structured surface on phase change intensity and cooling performance by setting the control group to include flat surfaces and structured surfaces with different pillars heights, which are $3 \mu \mathrm{m}, 5 \mu \mathrm{m}$ and $7 \mu \mathrm{m}$, but have the same size for pillar cross section area and the pitch between pillars (H3A33_25, H5A33_25 and H7A33_25);

Second, to understand the impact of pillar density array on phase change intensity and cooling performance by setting the control group including flat surfaces and structured surfaces with different size for pillars cross section area and pitches between pillars, so that the number of pillars per unit area is different, which are 9 pillars and 25 pillars per unit area. Pillar height and initial liquid volume are kept constant (H5A55_9 and H5A33_25);

Third, To recognize the impact of the above two factors on cooling effect, besides the enhancement caused by the additional solid-liquid interface area, two structures with the same 
interface area but different pillar heights pillar array densities are compared (H3A33_25 and H5A55_9).

Fourth, to realize the wettability arrangement influence on cooling effect, the pure hydrophilic structure and the structure with hydrophobic pillar top surface and hydrophilic substrate was compared (Hydrophilic_H5A33_25 and H5A33_25).

With the above four comparative studies we may conclude the criterions of favorable pillar dimension and pillar arrangement for phase change cooling. The purely hydrophilic flat surface was included in each comparison with structured surface for studying the improvement of phase change performance based on surface modifications.

\subsection{Numerical Simulation Method}

The numerical solution is obtained with the pressure-based finite volume scheme using a commercial CFD software, ANSYS 13.0 FLUENT. The terms in momentum and energy equations were discretized using the second-order upwind differencing scheme. Pressurevelocity coupling was achieved using the SIMPLE algorithm. Grids for all microstructures considered were generated using structured mesh. The geometric reconstruction interpolation scheme [57] was used for interface calculation. Transient time step sizes were chosen from $10^{-7}$ to $10^{-8} \mathrm{~s}$ for calculations. Convergence criteria are set as $10^{-3}$ for continuity, momentum and volume fraction equation, while that of the energy equation is set as $10^{-6}$. A structured mesh was used for the geometry with the smallest cell size equal to $0.5 \mu \mathrm{m}$.

The continuum method is used in this study by solving Navier-Stokes equations. Computational methods applied to such configurations give relatively fast and predictable 
results. The assumption of the continuous nature of the fluid enables the bulk fluid properties to be derived according to well defined theoretical or empirical relations. A non-dimensional parameter, the Knudsen number, is introduced to describe the validity of that approximation. It relates the average mean free path to the characteristic length as follows:

$$
K n=\frac{\lambda}{L}
$$

where $L$ is the characteristic length scale. $\lambda$ represents the mean free path of the fluid. It can be calculated as:

$$
\lambda=\frac{k_{B} T}{\sqrt{2} \pi d^{2} p}
$$

where $k_{B}$ is the Boltzmann constant in $\mathrm{J} / \mathrm{K}, T$ is the temperature in $\mathrm{K}, p$ is pressure, and $d$ is the diameter of the gas particles. The typical value of mean free path for air at ambient pressure and room temperature is $68 \mathrm{~nm}$ [58]. The character length of microstructure geometry ranges from $3 \mu \mathrm{m}$ to $5 \mu \mathrm{m}$. Thus the Knudsen number in this study is about 0.01 to 0.02 , which fulfills the condition $K n \leq 0.1$ that fluid can be treated as a continuous medium and described in terms of the macroscopic variables.

\subsection{Grid Independent Test}

The grid independence test has been made with different cells numbers as 290,156 for a fine mesh, 224,874 for the medium one and 152,555 for the coarse mesh. The bottom surface was examined with constant heat flux input as displayed in Fig. 3. The heat source temperature was examined with a constant heat flux boundary condition. The largest temperature difference 
between fine mesh and coarse mesh is $1.43 \mathrm{~K}$, while the maximum deviation between fine mesh and medium mesh is $0.14 \mathrm{~K}$. Because of the small deviation between the result of the medium mesh case and fine mesh case, the medium mesh was used for each case in this study.

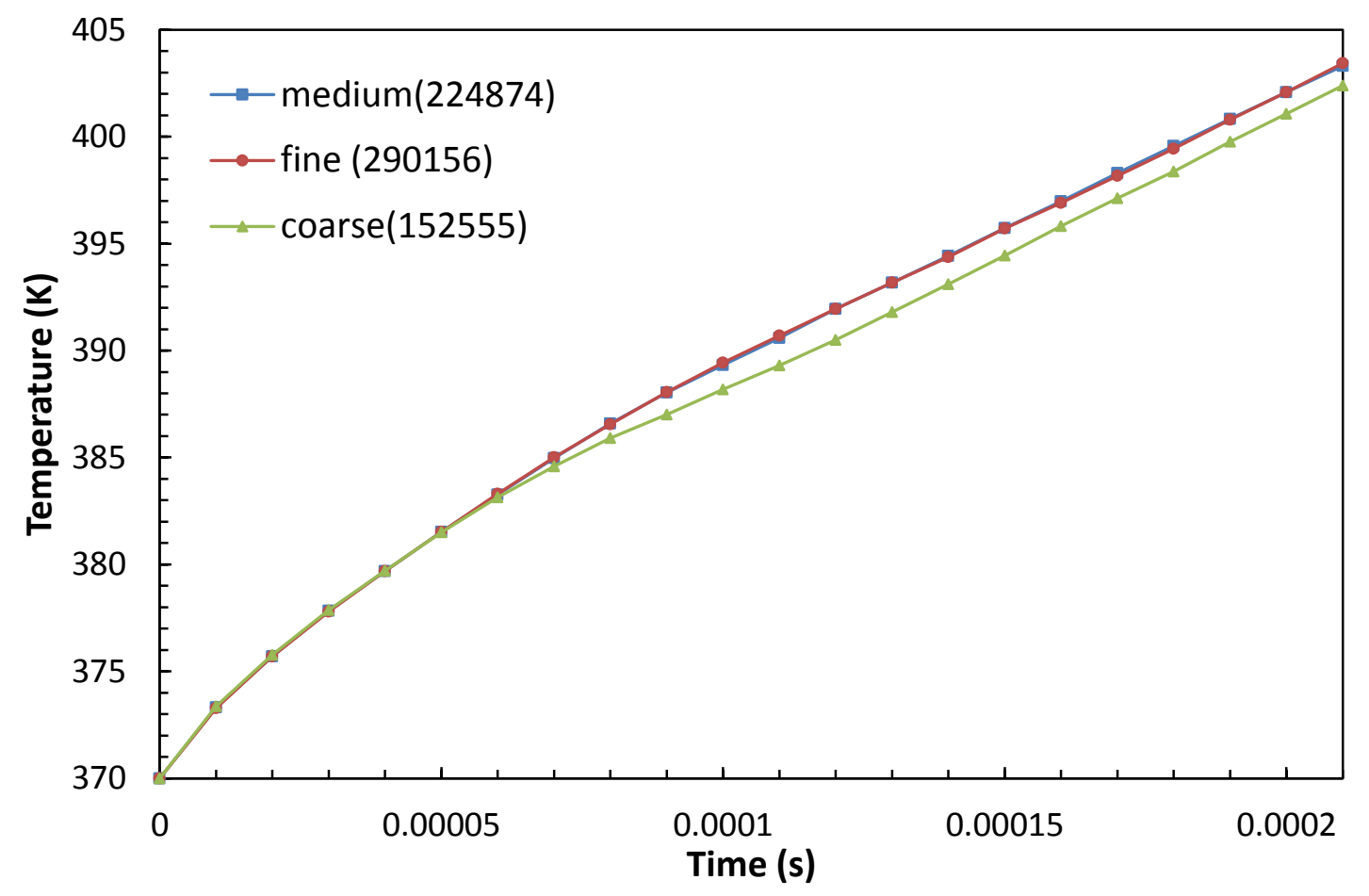

Fig. 3 Grid independent test for computational domain 


\section{CHAPTER 4: RESULTS AND DISCUSSION}

The discussion was divided into five parts. The emphasis for each part was summarized in Fig. 4. Part 3.1 discusses and compares the bubble dynamic behavior difference between flat surface and structured surface, as well as the fluid dynamic difference on structured surface with or without wettability texture. The comparative studies $3.2,3.3$ and 3.4 include two thermal boundary conditions, constant temperature and constant heat flux. The cases in 3.5 only take the constant heat flux boundary condition into account.

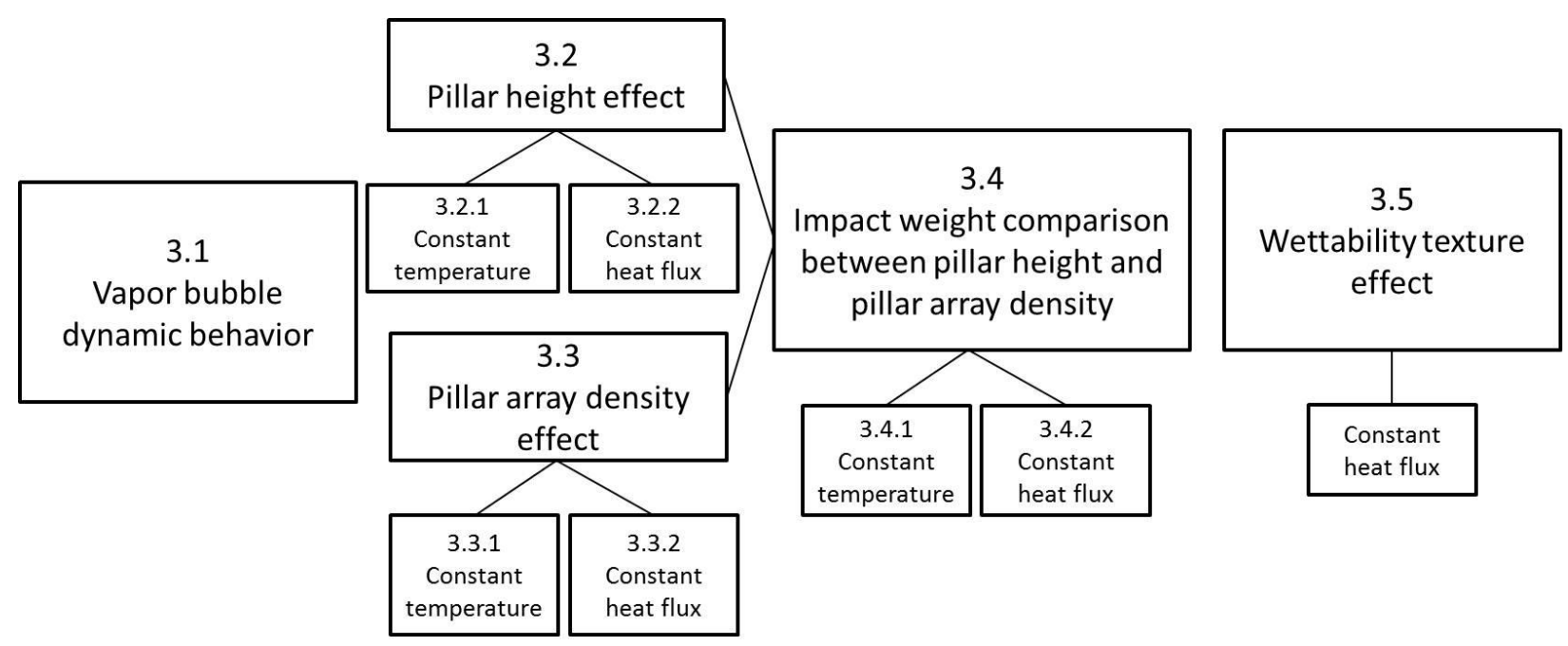

Fig. 4 Structure diagram of results and discussion

\subsection{Structure and Wettability Effect on Bubble Dynamic Behavior}

The bubble dynamic behavior on hydrophilic flat surface and wettability patterned flat surface are specified by Fig. 5 and Fig. 6. For hydrophilic flat surface, the generated bubble was distributed close to the substrate surface initially, while for wettability patterned flat surface most 
of the generated vapor appears on the hydrophobic spots in the beginning. The yellow color zones represent the hydrophobic surfaces and the red part is denoted as a hydrophilic surface. The liquid-vapor interface, including the bubble interface, was colored as blue. Consequently, the majority of the following coalescence is carried out between these bubbles in a horizontal direction. Because those horizontal merging processes can seldom introduce the upward momentum for bubble departure, the bubble departure frequency becomes relatively low and the average bubble departure size on a flat patterned surface becomes large, which indicates the flat surface cannot effectively trigger bubble departure.


Fig. 5 Boiling phenomena on hydrophilic flat surface 


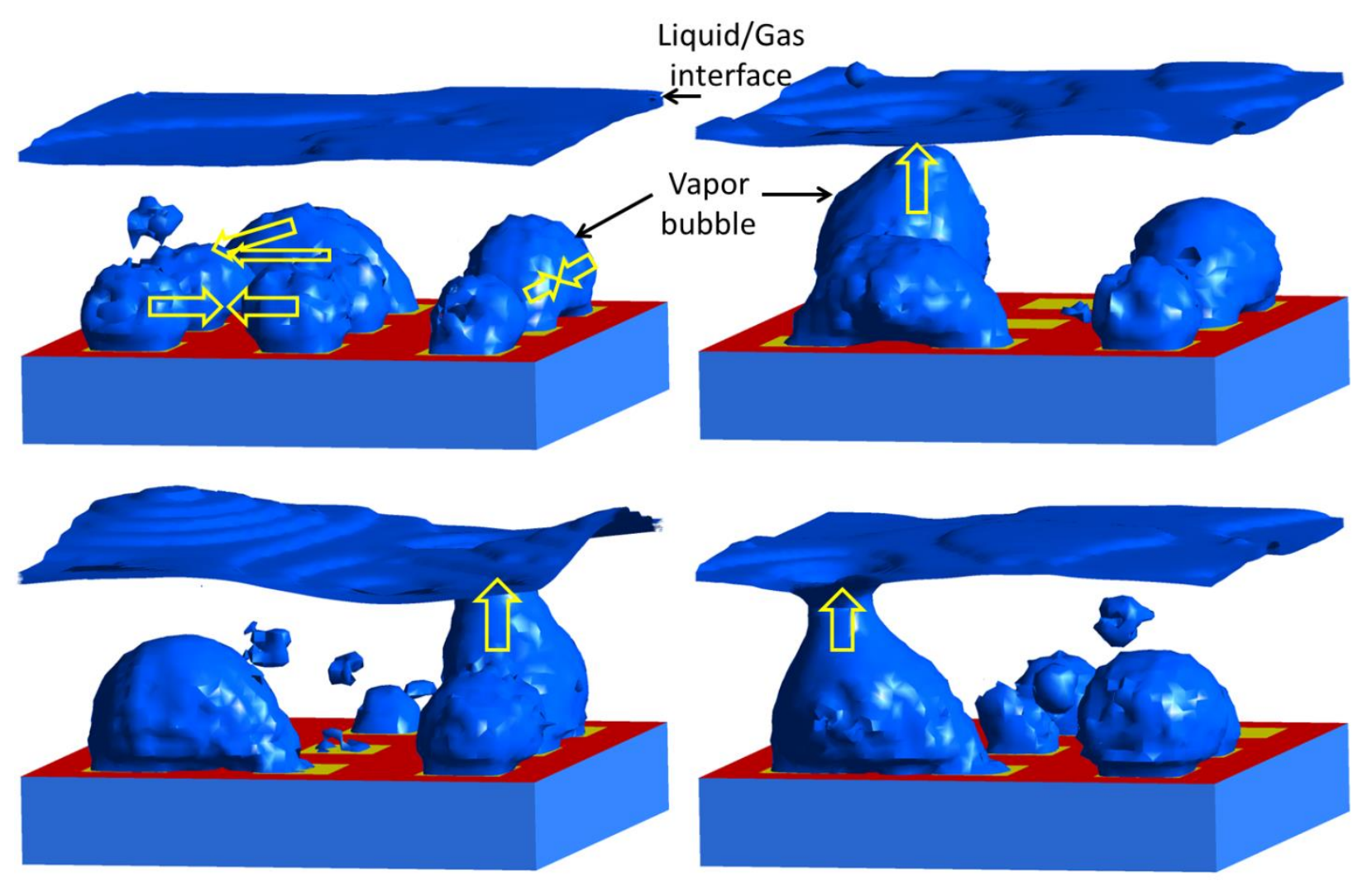

Fig. 6 Boiling phenomena on hydrophobic spot patterned flat surface

The velocity field variation accompanied with the bubble departure process on a flat patterned surface is revealed by Fig. 7. The phase change of water is primarily manifested as the accumulation of vapor on the hydrophobic spot. The direction of bubble motion is almost entirely kept to the horizontal direction during the merging process. The slight upward velocity, especially for the first merge, as the upper right figure depicts, is the result of the upward bubble centroid displacement. After experiencing the first merging, the newly composed bubble with larger volume has the inertia for keeping its stationary state when merging occurs with smaller bubbles. This is the reason for a significant decrease for both horizontal and vertical velocity during the second merging process. Bubble departure cannot be detected until the volume of the 
bubble is large enough for the upper interface to touch with the free surface. Beneficial vertical movement trend of bubble can seldom be initiated from the flat patterned surface, which limits bubble depature rate.

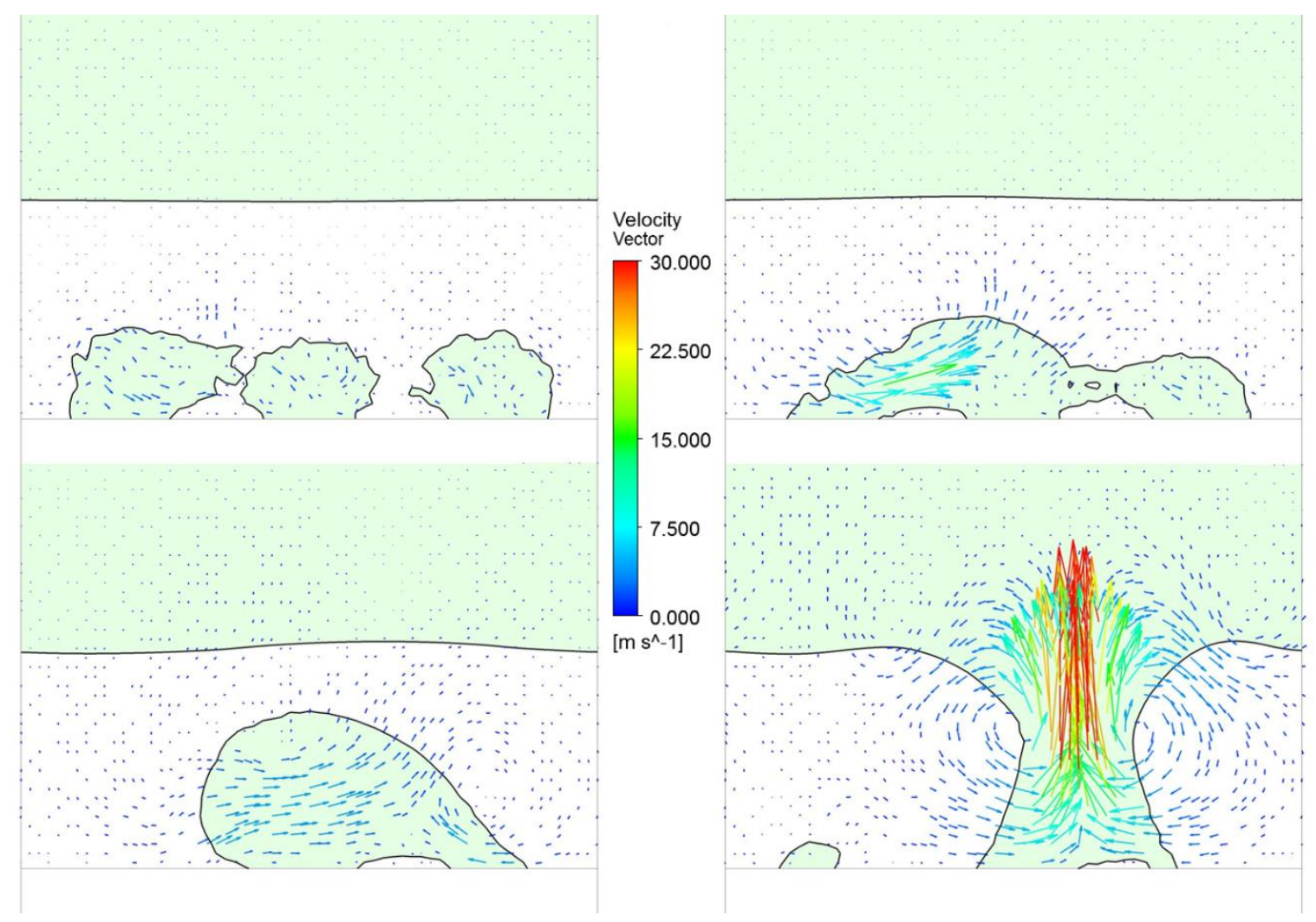

Fig. 7 Velocity field during bubble departure process on wettability patterned flat surface H0A55_9

The bubble dynamics on a hydrophilic structured surface are displayed in Fig. 8. Similar to the flat surface, most of the bubble motions induced by the merging process on a hydrophilic structured surface are in the horizontal direction. A large volume vapor bubble can be clearly observed after the merging and accumulation of small vapor bubbles. The opportunity for bubble 
departure on a hydrophilic structured surface is not high enough to prevent the large bubble from forming.
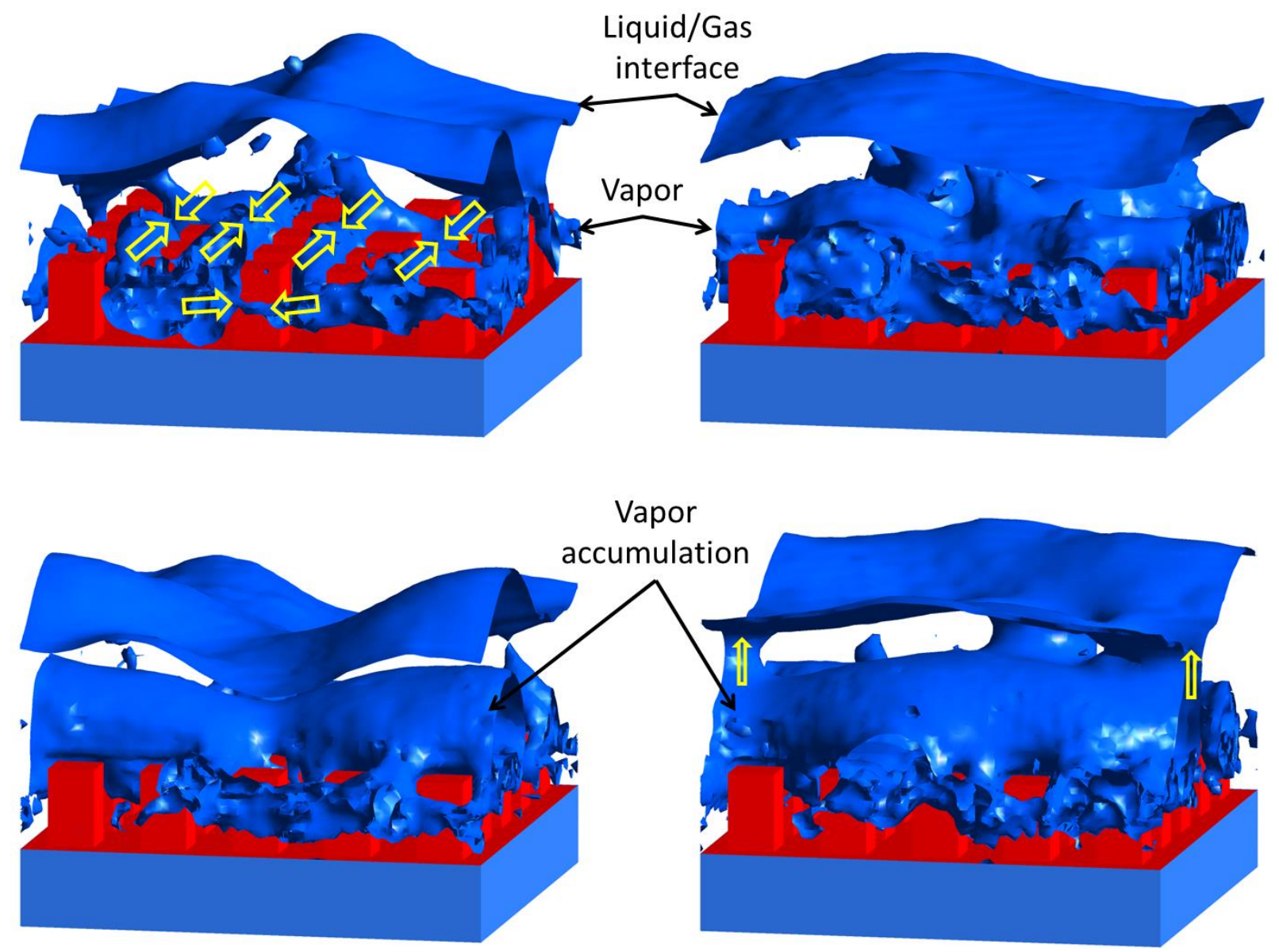

Fig. 8 Boiling phenomena on hydrophilic structured surface

Because of the relatively small volume of vapor bubbles and their low Bond number, the bubble motion is primarily produced by surface tension, and relevant induced dynamic inertia, rather than the buoyancy force. The Bond number can be expressed as:

$$
B o=\frac{\rho g L^{2}}{\sigma}
$$


For bubbles with radius smaller than $100 \mu \mathrm{m}$, the surface tension is over one thousand times higher than buoyancy force, thereby the significance of buoyancy force on bubble departure is diminished and the utilization of surface tension for bubble dynamic control was taken into account in this study.

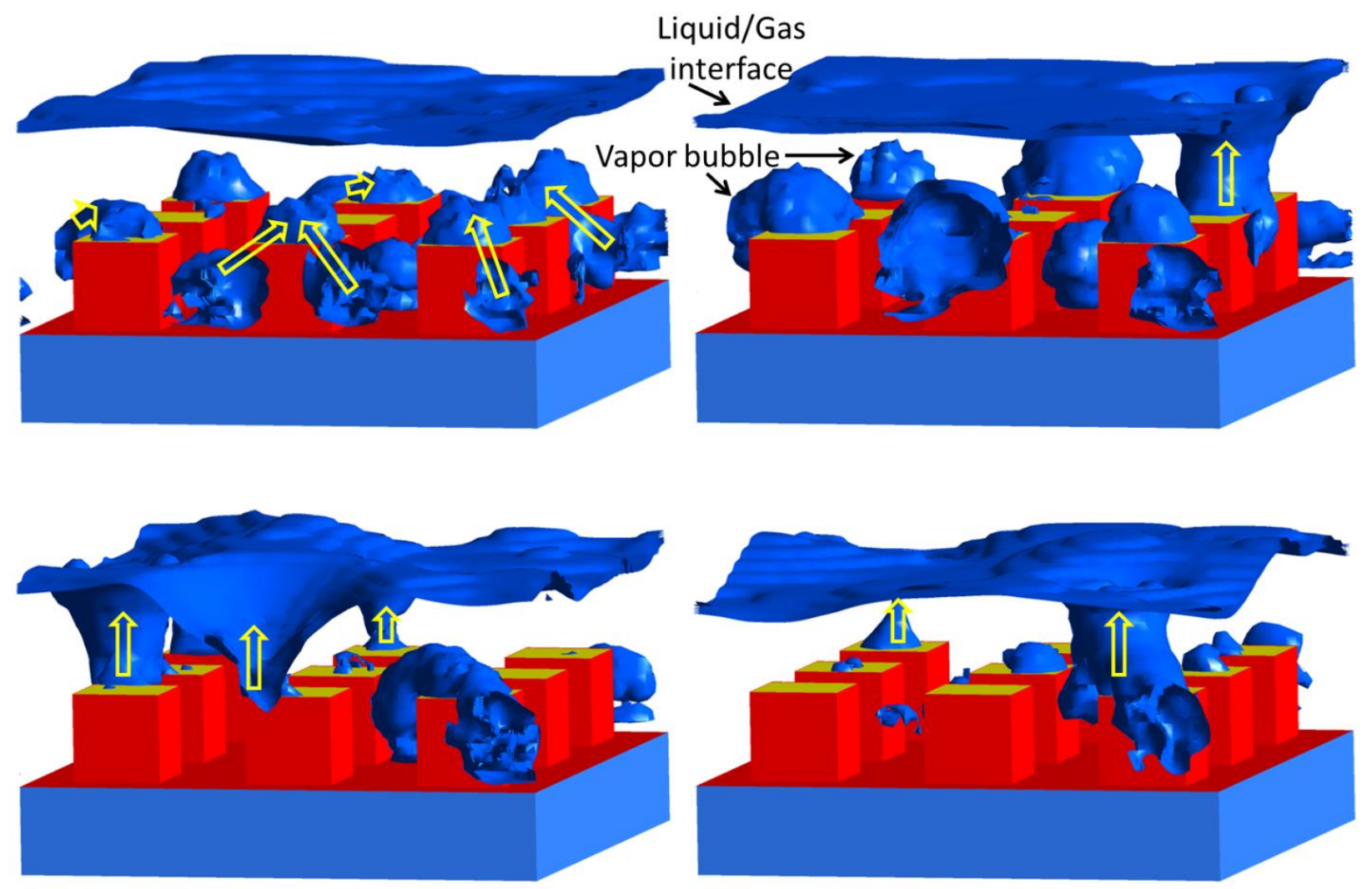

Fig. 9 Boiling phenomena on micro-structured surface with wettability gradient

The impact on bubble growth, coalescence and departure behavior by utilizing the structure combined with wettability gradient is displayed in Fig. 9. The objective for designing the combination of micro-pillar structure and wettability gradient is to build the surface tension gradient between hydrophobic pillar top surface and hydrophilic substrate, which enables those bubbles generated close to substrate surface to be pulled up. Most of the bubbles were generated 
close to the substrate surface because of the relatively higher temperature there with better bubble generation circumstance. At the same time, vapor bubbles were also generated at the pillar top surface due to its hydrophobic characteristic. From the yellow arrows in Fig. 9, we can observe the bubble motion trend in the next step. All the bubbles below the pillar top surface were pulled up by coalescence process with the bubble remaining on pillar top surface. This upward movement can not only bring bubbles closer to the liquid-gas interface, but also provides significant upward inertial momentum for triggering bubble departure, eventually causing frequent bubble departure phenomenon.

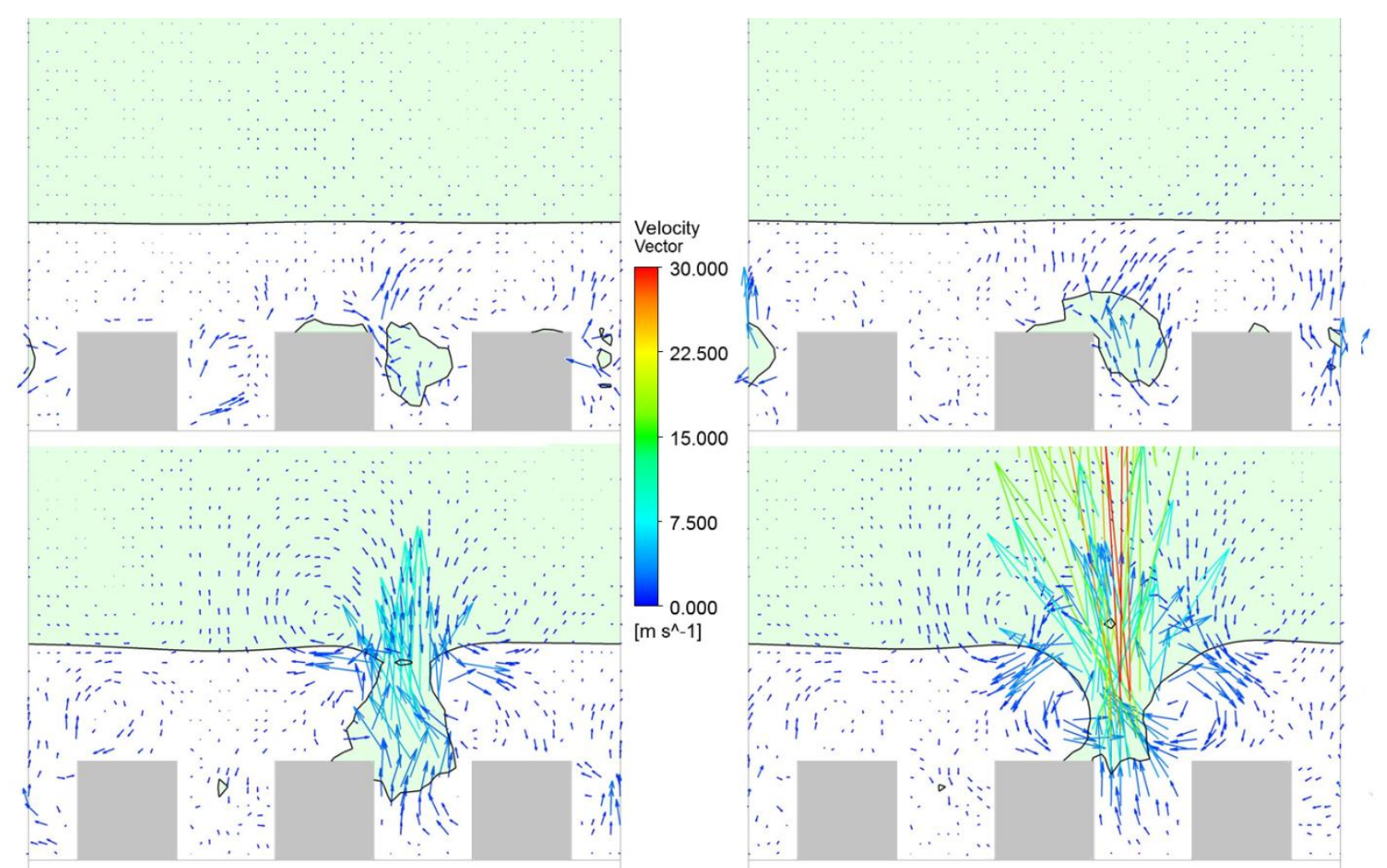

Fig. 10 Velocity field during bubble departure process on structured surface H5A55_9 
Fig. 10 demonstrates the velocity field variation and the bubble departure process. The bubble capturing process by hydrophilic pillar top surface induced a vertical velocity component in the upward direction for both vapor bubbles and the surrounding liquid. At the moment of departure, the velocity of the interface increases suddenly from $5 \mathrm{~m} / \mathrm{s}$ to a maximum of nearly $40 \mathrm{~m} / \mathrm{s}$, along with strong liquid vortices around the bubble. Liquid vortices conversely accelerate the detachment process. Therefore, it can be concluded from the comparisons shown in Figs. 5-10 that structured surfaces with engineered wettability have apparent improvement on bubble departure frequency.

\subsection{Comparative Studies for Structures with Vary Pillars Height}

\subsubsection{Constant Temperature Boundary Condition}

For understanding the solid-liquid heat dissipation effect of enhanced structured surface with various pillar heights, a constant temperature of $400 \mathrm{~K}$ was imposed on the bottom surface of the solid, and the whole domain was initialized with temperature equal to $370 \mathrm{~K}$. As time progresses, the mass-weighted average liquid temperature gradually increases from the initial temperature, which is presented by Fig. 11. The mass weighted average of a quantity $\phi$, which is fluid temperature here, can be represented as follows:

$$
\frac{\int \phi \rho d V}{\int \rho d V}=\frac{\sum_{i=1}^{n} \phi_{i} \rho_{i}\left|V_{i}\right|}{\sum_{i=1}^{n} \rho_{i}\left|V_{i}\right|}
$$

The results indicated that liquid temperature on structured surface with higher pillars respond faster with rapidly rising temperature, and always reach a relatively higher temperature at each time step within the simulation period. The liquid temperature's increasing rate reflects 
the solid-liquid heat transport efficiency of different surfaces. Remarkable heat dissipation from solid to liquid, such as the higher pillars structure in this case, leads to a better liquid thermal response to the heat source. For liquid-vapor phase change processes, higher liquid temperature is always accompanied by stronger phase change intensity, which can be represented by the parameter of mass transfer rate from liquid to vapor as specified in Fig. 12.

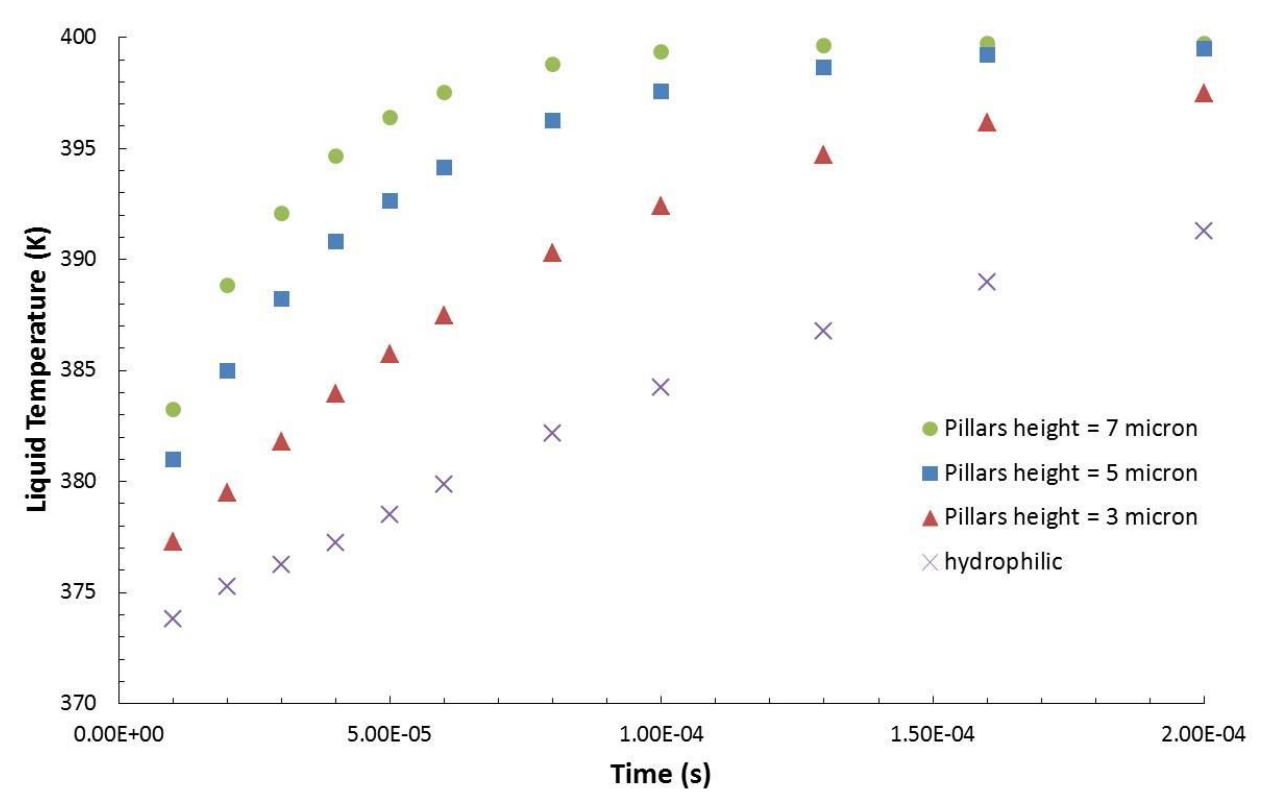

Fig. 11 Liquid temperature on surfaces with different pillar height $\left(T_{\mathrm{w}-\text { bottom }}=400 \mathrm{~K}\right)$

For understanding the average value of a certain quantity $\phi$ during a specific period, the time averaged value was calculated as follows:

$$
\frac{\int \phi d t}{\int d t}=\frac{\sum_{i=1}^{n} \phi_{i} t_{i}}{\sum_{i=1}^{n} t_{i}}
$$

The corresponding instant value of quantity $\phi$ at time $t_{i}$ is $\phi_{i}$. 


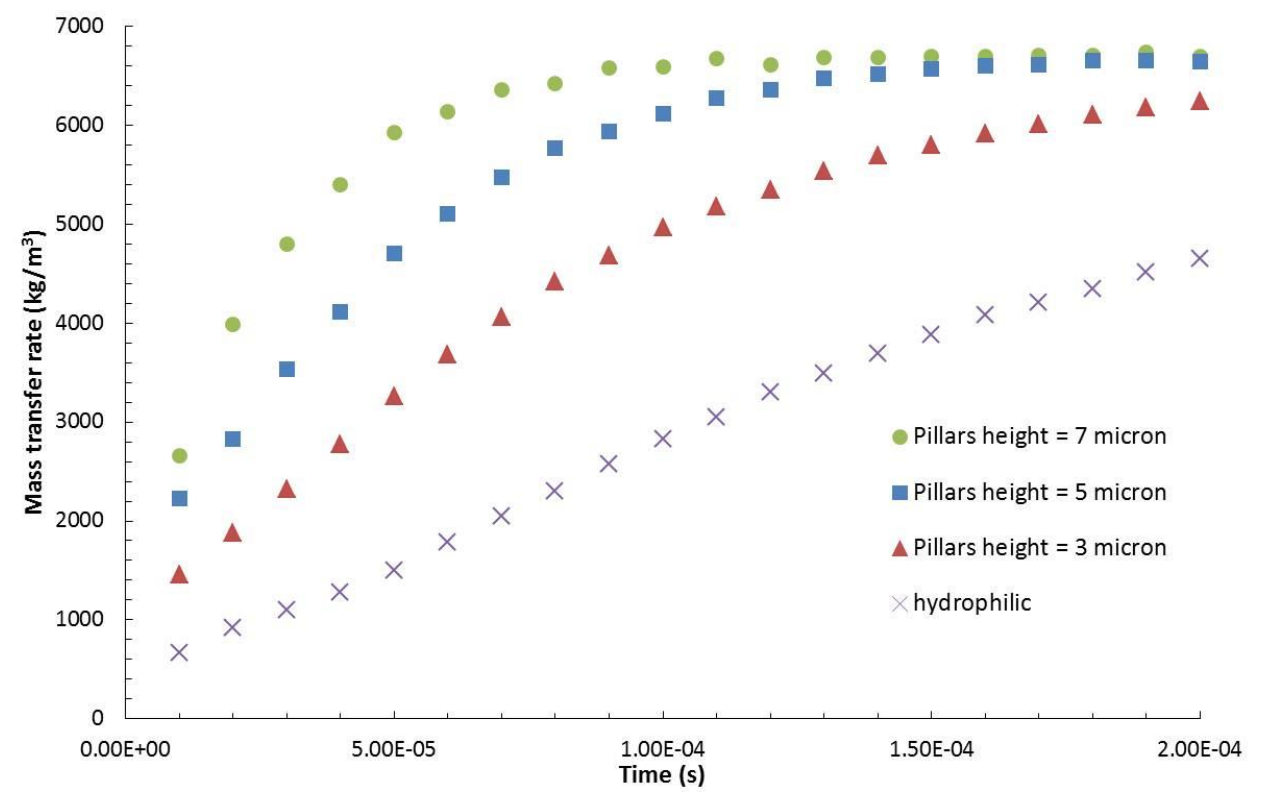

Fig. 12 Mass transfer rate on surfaces with different pillar height $\left(\mathrm{T}_{\mathrm{w} \text {-bottom }}=400 \mathrm{~K}\right)$

When compared with the time averaged mass transfer rate of the hydrophilic flat surface during $0.2 \mathrm{~ms}$, the rate of the structured surface with $3 \mu \mathrm{m}$ pillars, $5 \mu \mathrm{m}$ pillars and $7 \mu \mathrm{m}$ pillars was improved by $60.4 \%, 93.2 \%$ and $110.6 \%$ respectively. These results indicate the positive correlation between pillar height and phase change intensity within the initial $0.2 \mathrm{~ms}$ period. As time continues, the liquid temperature on the structured surface with $7 \mu \mathrm{m}$ pillars become stable, thus the liquid temperature difference and phase change intensity difference among all surfaces gradually decreases. The liquid temperature on the structured surface with 5 and $7 \mu \mathrm{m}$ pillars comes very close at the time $0.2 \mathrm{~ms}$, while due to slow heat dissipation from solid to liquid, the liquid temperature of the structured surface with $3 \mu \mathrm{m}$ pillars and of the flat surface are relatively lower. At the time $0.2 \mathrm{~ms}$, the solid-liquid heat dissipation from structured surfaces are all faster than the flat hydrophilic surface, and come with stronger phase change intensity. For example, the instant liquid-vapor mass transfer rate of liquid on $3 \mu \mathrm{m}, 5 \mu \mathrm{m}$ and $7 \mu \mathrm{m}$ pillar structures, at 
$0.2 \mathrm{~ms}$, are higher than the flat surface with $31.8 \%, 39.9 \%$ and $40.8 \%$ increases. The major reason for better solid-liquid heat dissipation achieved by the structured surface can be summarized due to the additional solid-liquid interface area. Therefore the liquid temperature can be heated faster and more uniformly with higher pillar structure.

\subsubsection{Constant Heat Flux Boundary Condition}

The constant heat flux boundary condition affords a more straightforward way to explore the thermal performance of different structures by examining the temperature rise of the heat source. According to the temperature contours in Fig. 13, as time progresses, the advantage of the structured surface on cooling can be clearly distinguished. At $0.1 \mathrm{~ms}$, the solid temperature of flat surface is above $394 \mathrm{~K}$, while the structured solid temperature is around $385 \mathrm{~K}$ to $388 \mathrm{~K}$. Lower solid temperature of structured surfaces reflected their heat transfer enhancement effect. Furthermore, the relatively lower solid temperature was always attained from the higher pillar surface at each time step. This result embodies the prominent heat dissipation capability induced by increasing the pillar height. In addition to the comparison of solid temperature among surfaces in Fig. 13, the phase change intensity of liquid over different structures can also be summarized. Because the liquid-vapor mass transfer rate of the fluid domain is directly dependent on the temperature of the liquid and gas phase, the higher average liquid temperature will bring a stronger mass transfer from liquid to vapor. 


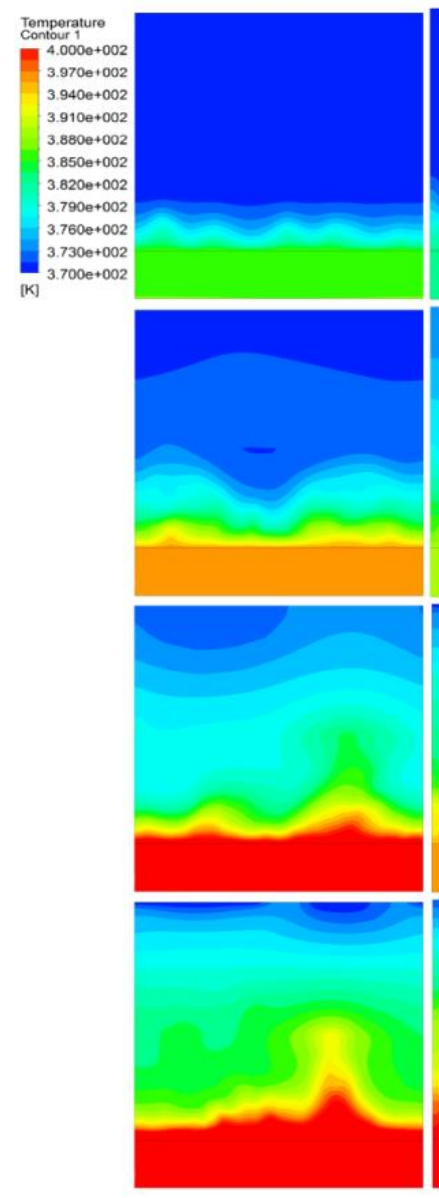

Hydrophilic

Flat Surface
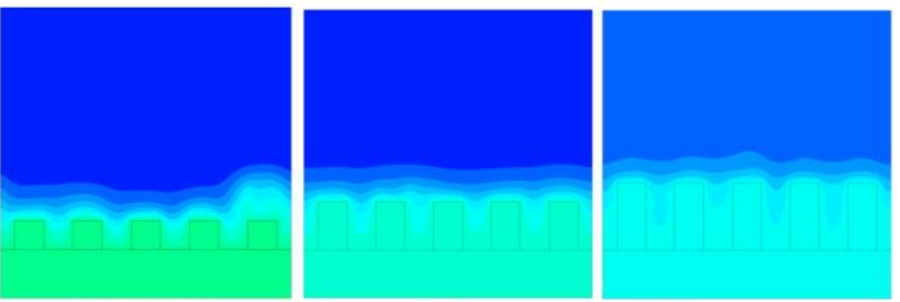

$0.05 \mathrm{~ms}$
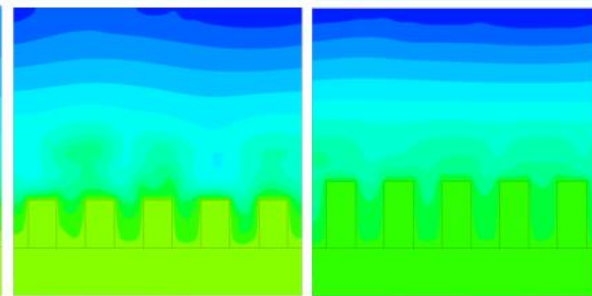

$0.1 \mathrm{~ms}$
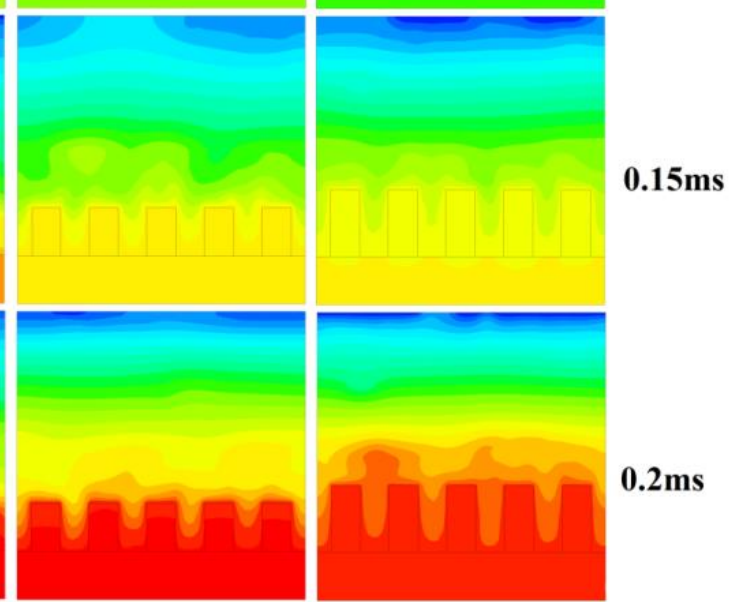

H5A33_25

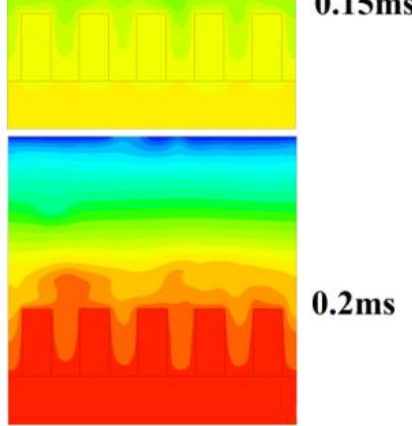

H7A33_25

Fig. 13 Temperature contours on surfaces with different pillar height

In Fig. 13, due to the limited solid-liquid interface area, only part of fluid domain on the flat surface was heated. The temperature of most of the fluid domain was kept at a relatively lower level. Thus the mass transfer rate on the flat surface was limited with less thermal energy dissipation in the form of latent heat. On the contrary, the fluid domain on the structured surface was heated faster and uniformly. Especially for structures with higher pillars, the sufficient solidliquid contact enables heat to be transported from the solid to fluid region farther from the 
substrate surface. Therefore the stronger phase change intensity along with better cooling effect can be expected on the higher pillar structured surface; the lower solid temperature on higher the pillar surface can be illustrated as well.

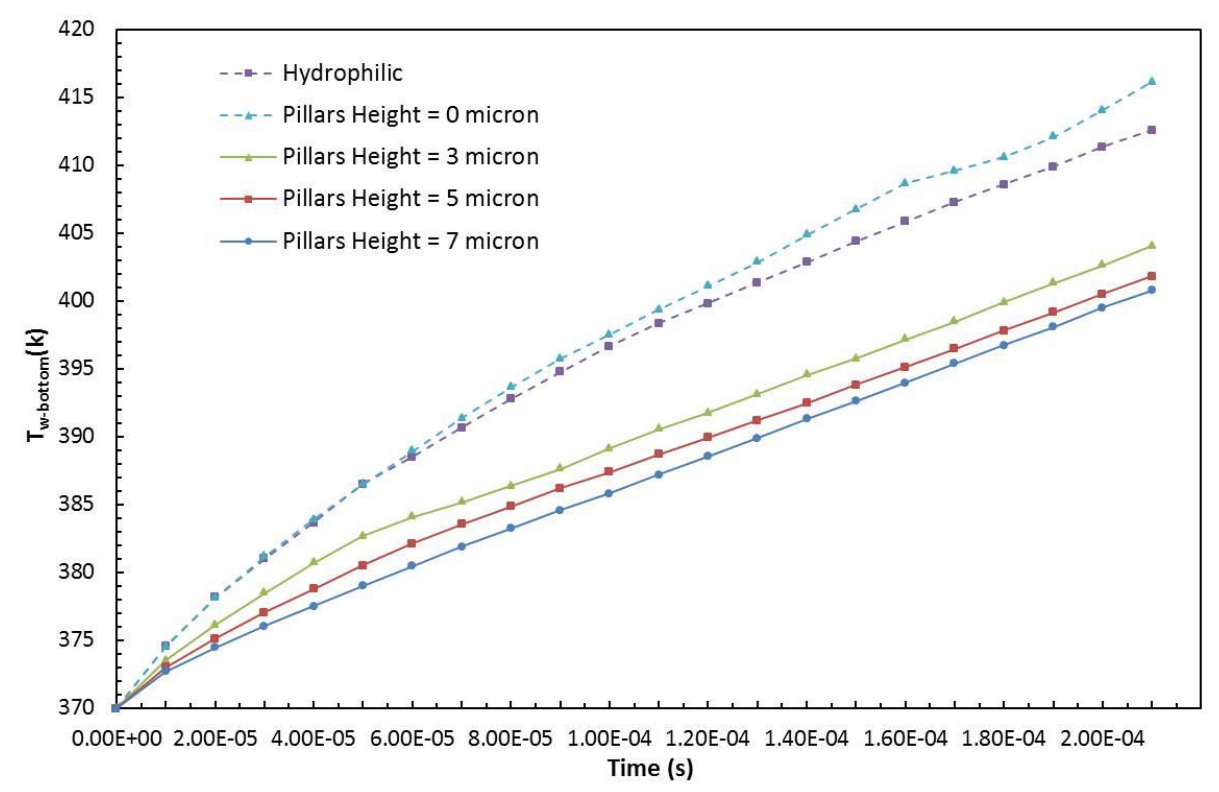

Fig. 14 Bottom surface temperature on surfaces with different pillar height

$$
\left(q^{\prime \prime}=700 \mathrm{~W} / \mathrm{cm}^{2}\right)
$$

As Fig. 14 demonstrated, after a heat flux $700 \mathrm{~W} / \mathrm{cm}^{2}$ was given to the bottom surface of the solid substrate, the corresponding bottom surface temperature increased gradually for structures with different pillar heights. The average bottom surface temperature of three structured surfaces during the first $0.21 \mathrm{~ms}$ period are $9.0 \mathrm{~K}$ lower than the flat surface with wettability pattern, and $7.7 \mathrm{~K}$ lower than the hydrophilic flat surface, which means the timeaveraged temperature rise was reduced by $31.9 \%$ with use of structured surface for the initial $0.21 \mathrm{~ms}$ when compared with using flat surfaces. At the time $0.21 \mathrm{~ms}$, the average temperature rise of the structured surface is $13.9 \mathrm{~K}$ lower than flat surfaces with wettability pattern and $10.4 \mathrm{~K}$ lower than the hydrophilic surface, which implies that the utilization of structured surface can 
decrease the heat source temperature rise to $72.7 \%$ of the value using flat surface. We notice that the wettability pattern on the flat surface did not improve the cooling effect. On the contrary, it decreases the thermal dissipation performance with higher heat source temperature than hydrophilic flat surface. One reason can be attributed to the large hydrophobic area in this pattern, which may easily cause bubble sticking on those lowly wettable spots. The presence of the bubble on the hydrophobic surface blocked the solid-liquid contact, and increased the difficulty of solid-liquid heat dissipation.

For comparison among structured surfaces, revealed by Fig. 14, surfaces with higher pillars structure have noteworthy cooling effect and relatively lower bottom surface temperature. The average temperature rise for initial $0.21 \mathrm{~ms}$ of the $7 \mu \mathrm{m}$ pillar surface is $1.2 \mathrm{~K}$ and $2.9 \mathrm{~K}$ lower than the surface with $5 \mu \mathrm{m}$ and $3 \mu \mathrm{m}$ pillars. For the heat source temperature at $0.21 \mathrm{~ms}$, lowest temperature $400.8 \mathrm{~K}$ was achieved by $7 \mu \mathrm{m}$ pillar surface, while the heat source temperature on the $5 \mu \mathrm{m}$ pillar surface and $3 \mu \mathrm{m}$ pillar surface are $1 \mathrm{~K}$ and $3.3 \mathrm{~K}$ higher respectively.

Because of the prominent solid-liquid heat transfer of the higher pillar structure discussed before, the phase change intensity on the higher pillar surface was enhanced, as the mass transfer rate curves represented in Fig. 15. Higher phase change rate implies more thermal energy was taken away in the form of latent heat, which explained the lower heat source temperature achieved by the structured surface and higher pillar surface. The time averaged mass transfer rates within $0.21 \mathrm{~ms}$ for two flat surfaces were enhanced by $3 \mu \mathrm{m}$ pillar, $5 \mu \mathrm{m}$ pillar and $7 \mu \mathrm{m}$ pillar surfaces with $23.1 \%, 35.3 \%$ and $42.4 \%$ increases respectively. As time progress, the mass transfer rate was increasing as the temperature of liquid on the structured surface was heated. The phase change intensity on the structured surface is always higher than the flat surface, and the difference gradually becomes noticeable after $0.03 \mathrm{~ms}$. At time $0.21 \mathrm{~ms}$, the mass transfer rate 
for hydrophilic surface and flat surface with wettability pattern are $4,930.3 \mathrm{~kg} / \mathrm{m}^{3} \mathrm{~s}$ and $4,806.5$ $\mathrm{kg} / \mathrm{m}^{3} \mathrm{~s}$, respectively. On the basis of mass transfer rate of hydrophilic surface, the structured surface with $3 \mu \mathrm{m}$ pillars, $5 \mu \mathrm{m}$ pillars and $7 \mu \mathrm{m}$ pillars improved the phase change intensity by $13.7 \%, 23.1 \%$ and $26.9 \%$ at time $0.21 \mathrm{~ms}$.

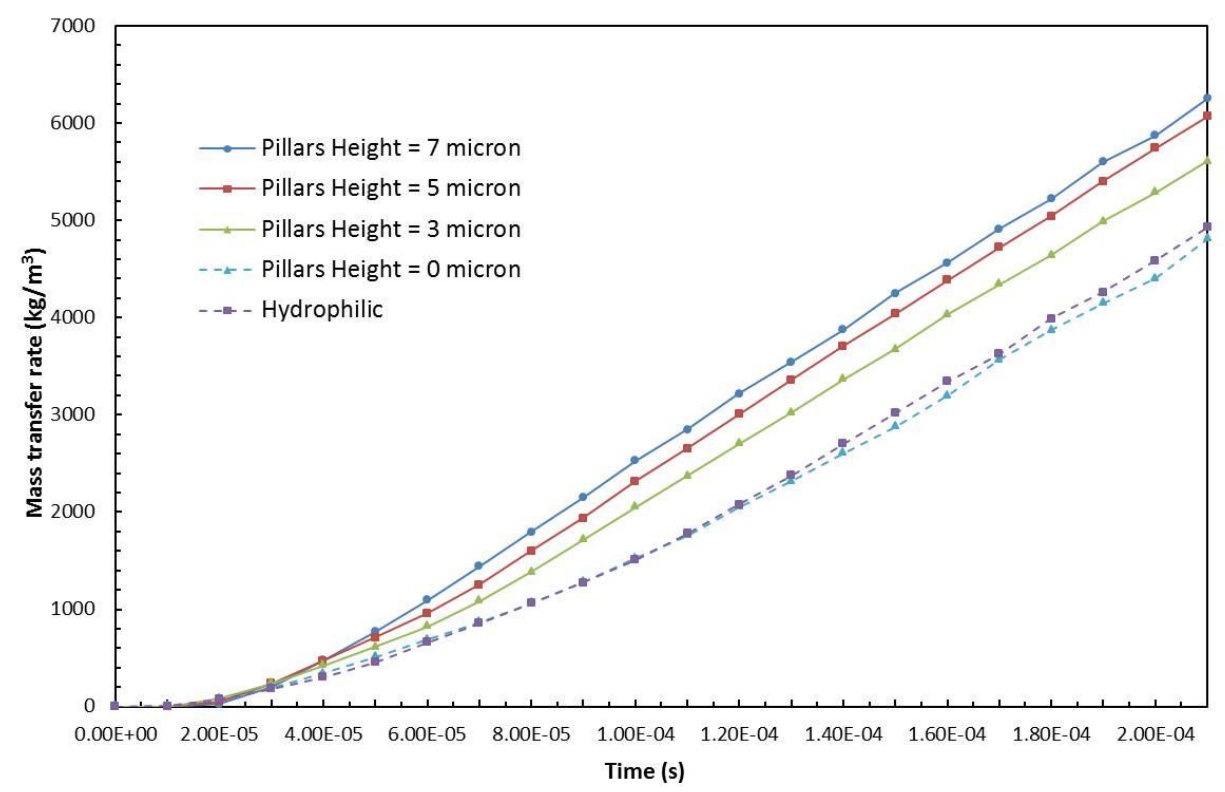

Fig. 15 Mass transfer rate on surfaces with different pillar height $\left(q_{w}{ }^{\prime \prime}=700 \mathrm{~W} / \mathrm{cm}^{2}\right)$

The heat transfer coefficient is a typical parameter for calculating the heat transfer process including convection and phase change, which is the proportionality coefficient between solid-liquid interface heat flux and solid-liquid temperature difference. The heat transfer coefficient here was calculated with the following equation:

$$
h=\frac{q_{w-i}^{\prime \prime}}{T_{w-i}-T_{l}}
$$

Where $q_{w-i}^{\prime \prime}$ indicates the heat flux across the solid-liquid interface, the corresponding temperature of solid-liquid interface is denoted by $T_{w-i}$, and the liquid temperature is $T_{l}$. Thus 
the heat transfer coefficient can be used as an indication to evaluate the energy transportation efficiency from solid to liquid.

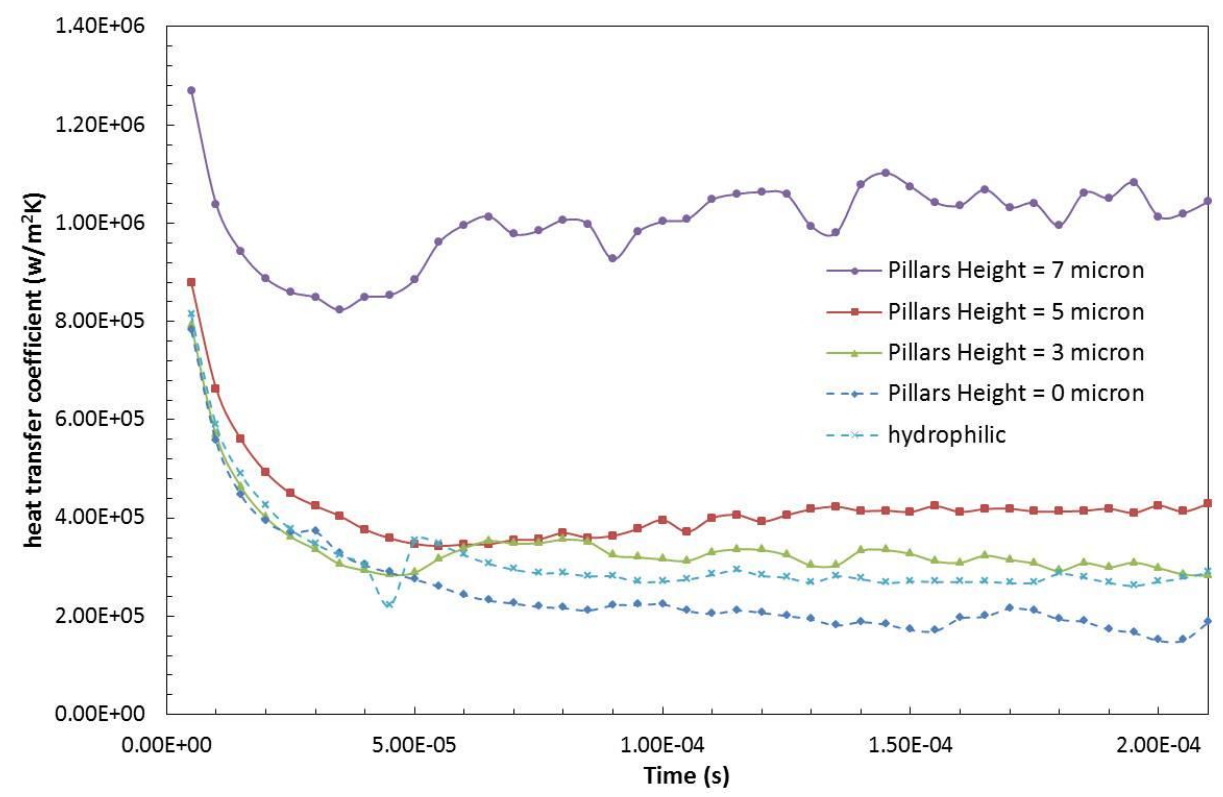

Fig. 16 Heat transfer coefficient on surfaces with different pillar height

$$
\left(\mathbf{q}_{w}{ }^{\prime \prime}=700 \mathrm{~W} / \mathrm{cm}^{2}\right)
$$

Higher solid-liquid interface heat flux and lower solid-liquid temperature difference creates a higher heat transfer coefficient, just as the value of higher pillar surface demonstrated in Fig. 16. Because of the fluctuation of the heat transfer coefficient, the instant value may not be able to reflect the real heat dissipation effect of those surfaces. Thus we compare the heat transfer coefficient by using the time averaged value during $0.21 \mathrm{~ms}$. Similar to former results, the flat surface with wettability patterns has the worst thermal performance with the lowest heat transfer coefficient, the corresponding time averaged value is $250,941 \mathrm{~W} / \mathrm{m}^{2} \mathrm{~K}$, while the value for hydrophilic flat surface is $25.6 \%$ higher than the patterned flat surface. The heat transfer enhancement by the structured surface can also be recognized. The time averaged heat transfer 
coefficient of structured surface with $3 \mu \mathrm{m}, 5 \mu \mathrm{m}$ and $7 \mu \mathrm{m}$ pillars are $8.4 \%, 33.2 \%$ and $217.7 \%$ higher than the hydrophilic surface, respectively. For the structured surface with $7 \mu \mathrm{m}$ pillars this is especially true, because of the excellent thermal dissipation from solid to liquid, relatively smaller temperature gaps between solid and liquid can be achieved, thus the heat transfer coefficient of structured surface with $7 \mu \mathrm{m}$ pillars is significantly higher than others, revealing excellent heat dissipation capacity.

\subsection{Comparative Studies for Structures with Various Pillar Array Density 4.3.1 Constant Temperature Boundary Condition}

Comparative study for pillar array density with constant temperature heat source $(400 \mathrm{~K})$ was made on micro-pillar structures containing 9 pillars and 25 pillars, for recognizing the solidliquid heat dissipation effect. Dense pillar array contains more pillars with a slimmer shape, which is $5 \mu \mathrm{m}$ high by $3 \mu \mathrm{m}$ long by $3 \mu \mathrm{m}$ wide, while sparse pillar array in this study is composed of pillars with dimension $5 \mu \mathrm{m}$ high by $5 \mu \mathrm{m}$ long by $5 \mu \mathrm{m}$ wide. The pillar height and initial liquid volume of both cases were kept the same.

The calculation result showed by Fig. 17 indicates the liquid on structures with 25 pillars was heated faster than the liquid on structures with 9 pillars, from the beginning to $0.2 \mathrm{~ms}$, so that the liquid temperature on a dense pillar array is always higher. Again, the liquid temperature response on structured surfaces was far quicker than the liquid temperature response on flat surfaces. Thus we may draw the same conclusion that thermal energy can be more easily transported from solid to liquid phase by structured surface, especially the structure containing dense pillar array with slim pillars. 


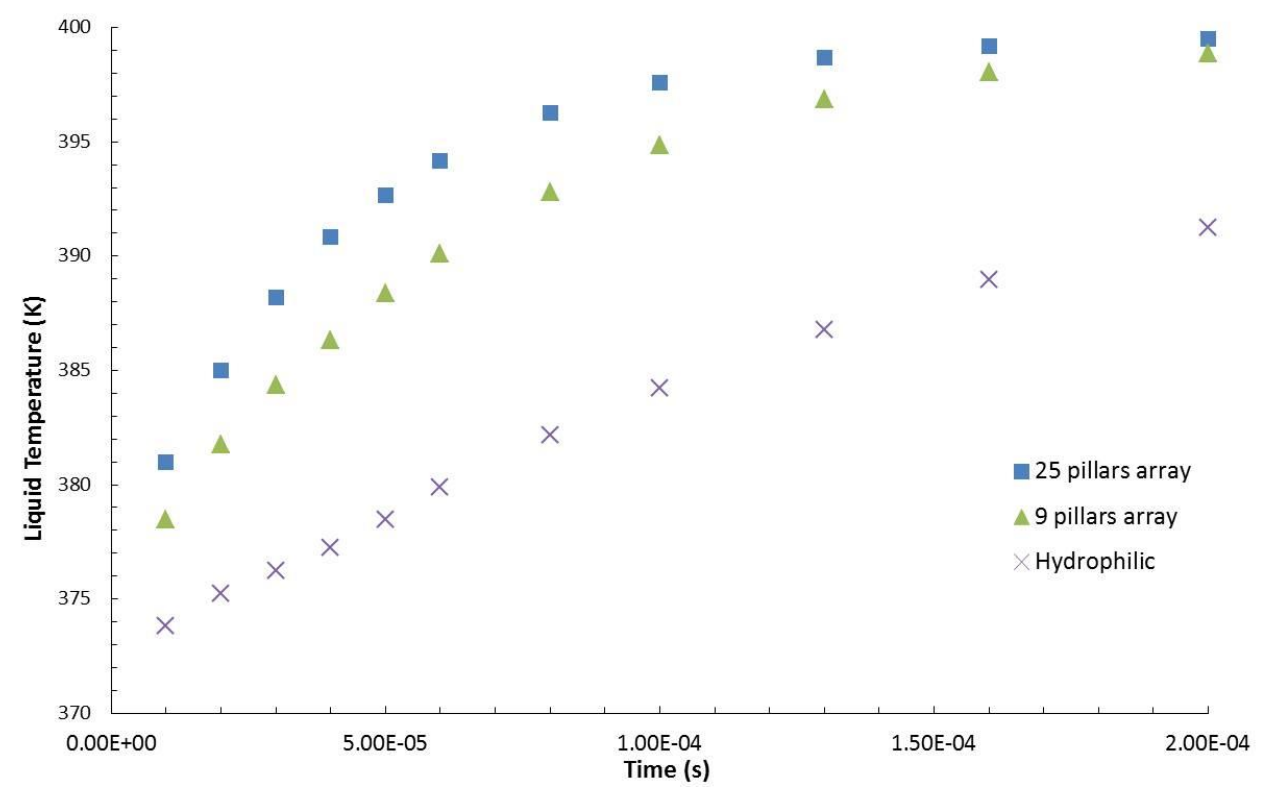

Fig. 17 Liquid temperature on surfaces with different pillar array density $\left(\mathrm{T}_{\mathrm{w}-\mathrm{bottom}}=\mathbf{4 0 0 K}\right)$



Fig. 18 Mass transfer rate on surfaces with different pillar array density $\left(\mathrm{T}_{\mathrm{w}-\mathrm{bottom}}=\mathbf{4 0 0 K}\right)$ 
With faster temperature increase and higher temperature at each moment, the stronger liquid-vapor mass transfer for the phase change process can be achieved at each time step, which was presented by the mass transfer variation curve in Fig. 18. The time averaged mass transfer rates during $0.2 \mathrm{~ms}$ of 9 pillar and 25 pillar structured surfaces were $76.7 \%$ and $93.2 \%$ higher than the hydrophilic flat surface respectively. At $0.2 \mathrm{~ms}$, the mass transfer rate of the 25 pillar structure and 9 pillar structure are $39.9 \%$ and $37.7 \%$ higher than the instant mass transfer rate of hydrophilic flat surface. The solid-liquid heat transfer enhancement on dense array surface H5A33_25 can be attributed to its larger solid-liquid interface area rather than sparse array surface H3A33_25.

\subsubsection{Constant Heat Flux Boundary Condition}

Fig. 19 demonstrated the temperature contours on surfaces with different pillar array densities. The solid temperature of the dense array surface was maintained at a lower extent at each time step. The excellent cooling performance of dense pillar array structures can therefore be easily observed. For understanding the liquid domain phase change intensity it is necessary to know the average liquid temperature. For both structures H5A55_9 and H5A33_25, the heat was conducted to the same distance away from the substrate surface, as a result of the same pillar height, at each time step exhibited in Fig. 19. However, in the dense array surface, more widely distributed pillars shorten the horizontal distance between pillars. The liquid around the pillars in the dense array surface can be heated faster and more uniformly. While for sparse array surface, the higher liquid temperature gradient around the pillars can be observed, which presents the uneven heating phenomena of liquid domain between pillars. The stronger phase change 
intensity along with better cooling effect can be expected on the dense pillar array structured surface, the lower solid temperature on the dense array surface can be illustrated as well.

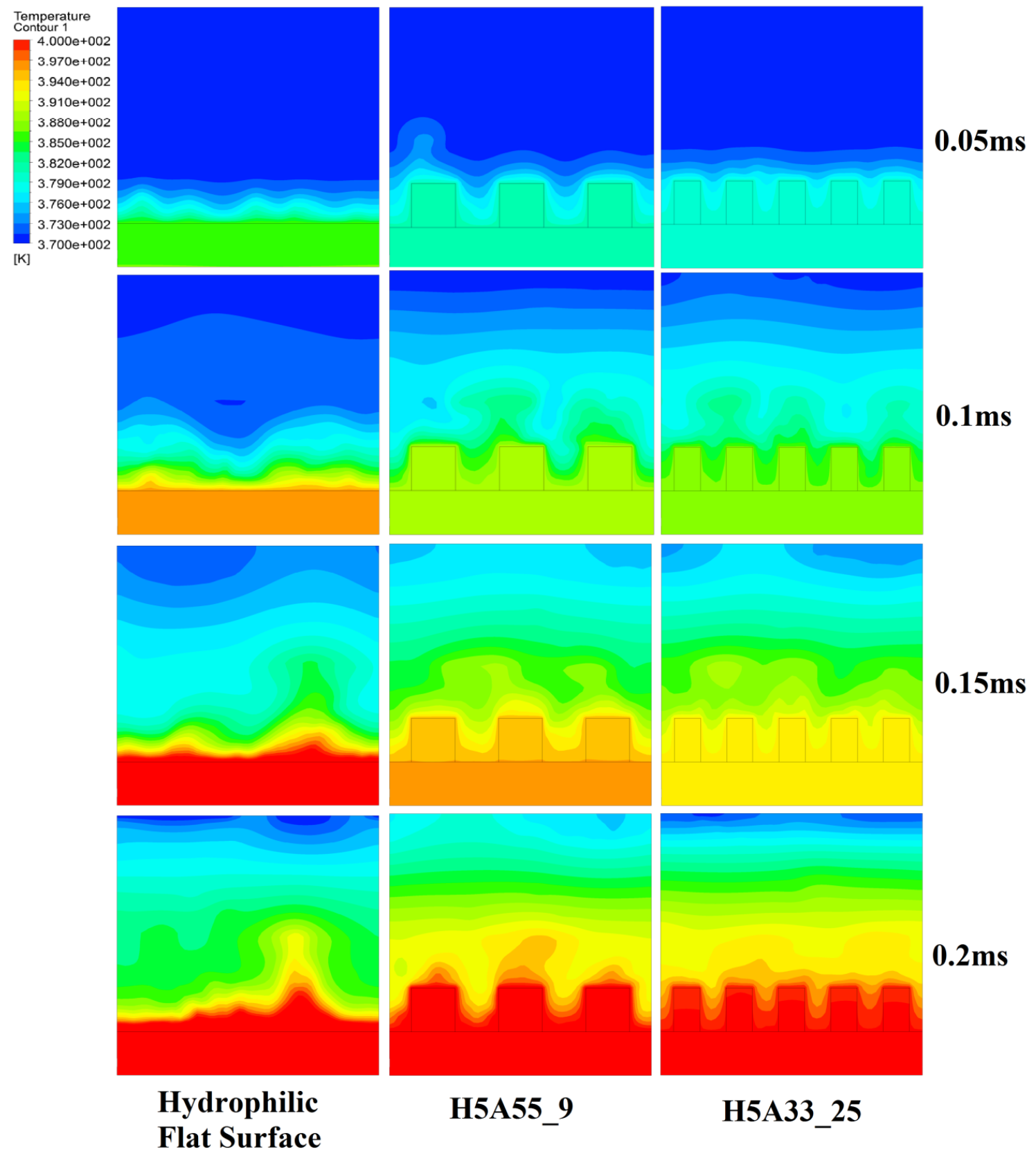

Fig. 19 Temperature contours on surfaces with different pillar array density 
After being heated with a constant heat flux $700 \mathrm{~W} / \mathrm{cm}^{2}$, the bottom surface temperature variation of structures with different pillar array densities can be found from Fig. 20; the temperature difference between the structured surface and flat surface can still be clearly observed.



Fig. 20 Bottom surface temperature on surfaces with different pillar array density $\left(q_{w}{ }^{\prime \prime}=700 W / \mathrm{cm}^{2}\right)$

The time averaged bottom surface temperature of the flat surface during $0.21 \mathrm{~ms}$ reaches 395.4K, while the temperature for 25-pillar surface and 9-pillar surface are $7.9 \mathrm{~K}$ and $6.3 \mathrm{~K}$ lower than the flat one respectively, lower than the total temperature rise of flat surface by $31.1 \%$ and $24.8 \%$, respectively, thus the superior cooling effect induced by the structured surface with dense pillar array can be examined. The instantaneous bottom surface temperature at $0.21 \mathrm{~ms}$ for the flat surface, 9-pillar surface and 25-pillar surface are 412.6K, 403.4K and 401.8K, respectively. 
Compared with flat hydrophilic surface, cooling by structured 25-pillar surface can reduce the total temperature rise by $25.4 \%$ at $0.21 \mathrm{~ms}$, and for the 9-pillar surface, the percentage of reduced amount of total temperature rise is $21.6 \%$.

Similarly, the lower temperature of the heated surface was maintained because the microstructure surface enhanced phase change intensity as shown in Fig. 21. The higher liquidvapor mass transfer rate can afford faster thermal energy dissipation. The time averaged mass transfer rates of structured surfaces with 25 pillars and 9 pillars were improved $33.9 \%$ and $27.6 \%$ based on the value of hydrophilic flat surface during the initial $0.21 \mathrm{~ms}$. The instant value for mass transfer rate at time $0.21 \mathrm{~ms}$ was enhanced by $23.1 \%$ and $20.4 \%$ with the 25 -pillar surface and 9-pillar surface respectively.

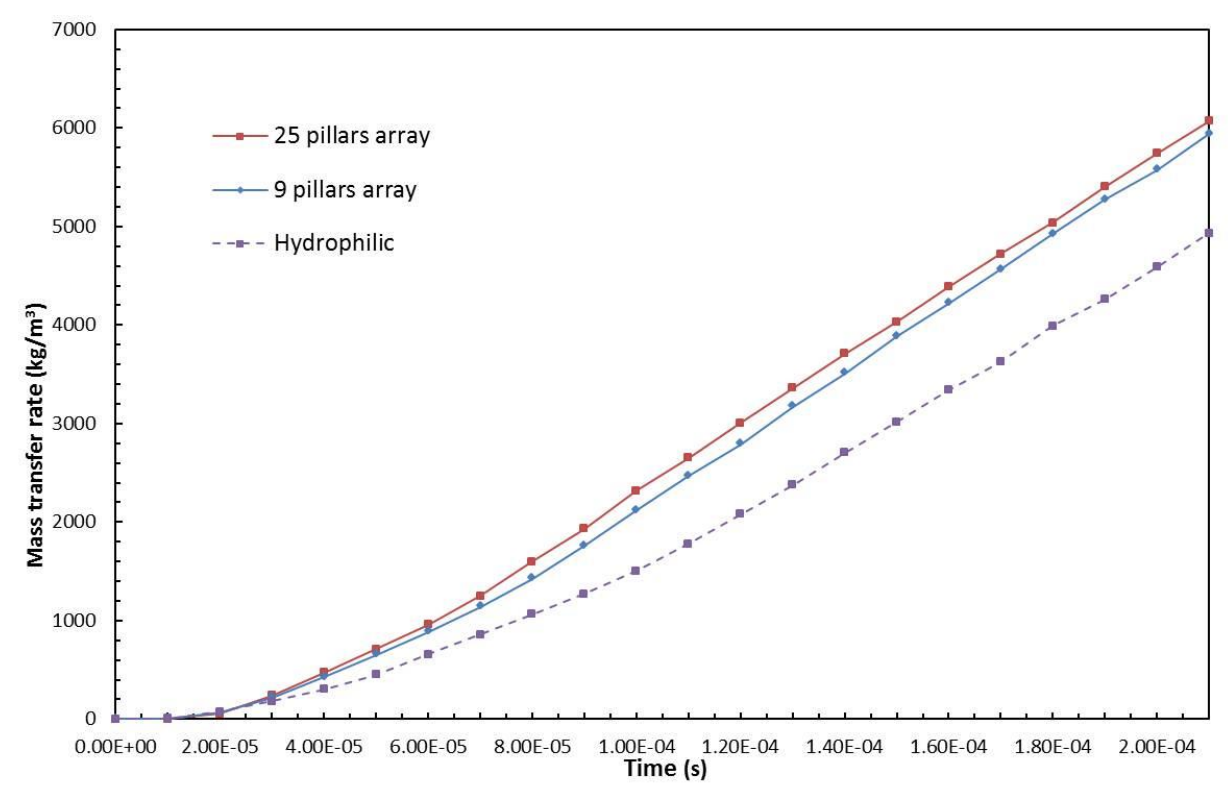

Fig. 21 Mass transfer rate on surfaces with different pillar array density

$$
\left(q_{w} "=700 W / \mathrm{cm}^{2}\right)
$$


The greater thermal performance of the dense pillar array structure was also validated by the heat transfer coefficient curve displayed in Fig. 22. From the beginning to $0.2 \mathrm{~ms}$, the time averaged heat transfer coefficient for the hydrophilic surface was improved by $33.2 \%$ and $18.8 \%$ with the utilization of structured surfaces containing 25 pillars and 9 pillars respectively.



Fig. 22 Heat transfer coefficient on surfaces with different pillar array density $\left(q_{w}{ }^{\prime \prime}=700 W / \mathrm{cm}^{2}\right)$

\subsection{Comparative Study for Structures with Same Extra Solid-Liquid Interfacial Area 4.4.1 Constant Temperature Boundary Condition}

For both the comparative studies discussed above, one reason for the heat transfer enhancement of the structured surfaces is attributed to the increasing solid-liquid interface area. For understanding the impact of pillar height and pillar array density on thermal dissipation, the structure H3A33_25 and H5A55_9 with the same solid-liquid interface area were selected to be compared. The structure H5A55_9 has higher pillars and a sparser pillar array, while structure H3A33_25 has a denser pillar array and shorter pillars. 


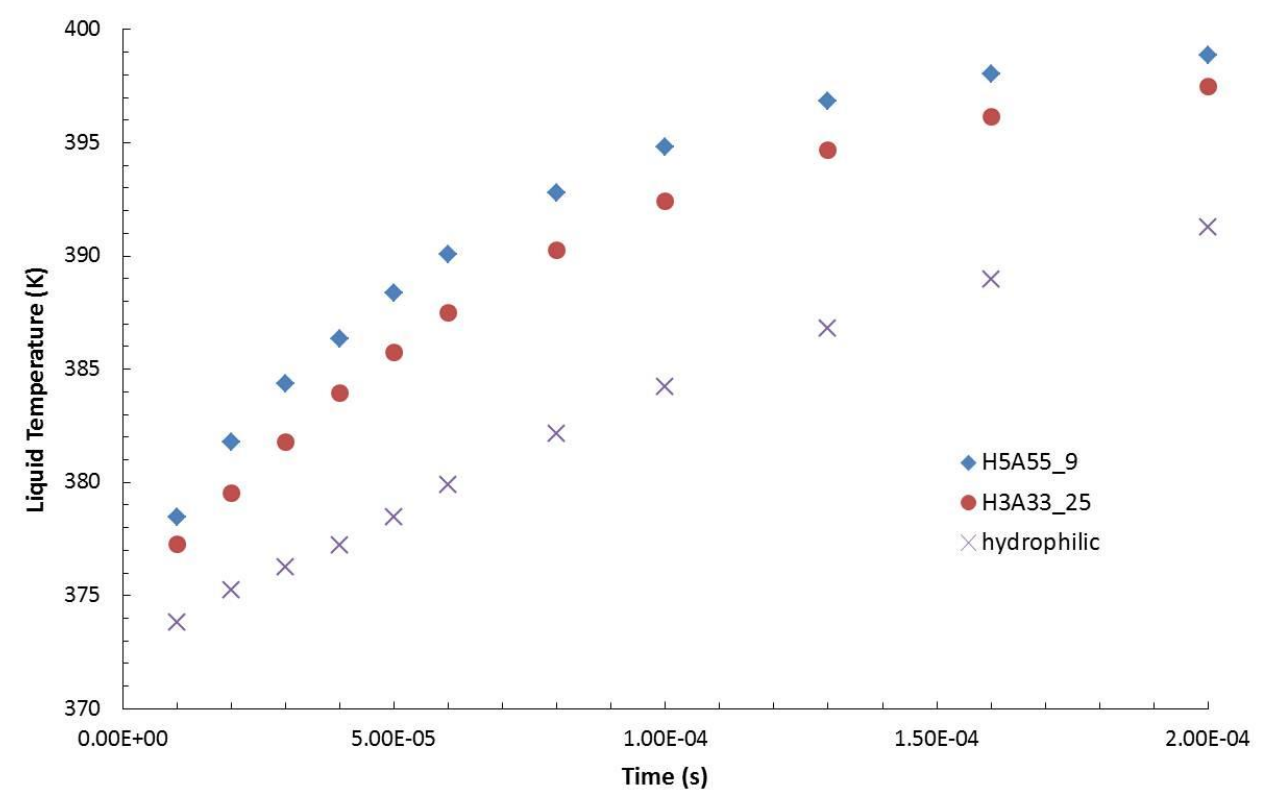

Fig. 23 liquid temperature on surfaces for comparison of impact between pillar height and pillar array density $\left(T_{\mathrm{w}-\mathrm{bottom}}=400 \mathrm{~K}\right)$

Fig. 23 exhibits the liquid temperature variation on structures H3A33_25 and H5A55_9 when the domain was heated by a constant temperature of $400 \mathrm{~K}$, and the liquid-vapor mass transfer variation under the same condition was shown by Fig. 24. The liquid on structure H5A55_9 presents a relatively faster temperature response, higher liquid temperature and stronger phase change intensity during $0.2 \mathrm{~ms}$. Based on the time averaged mass transfer rate of the hydrophilic surface, the structure H3A33_25 enhanced the rate by $60.4 \%$ and the structure H5A55_9 improved the rate by $76.7 \%$. The instant mass transfer rate on the hydrophilic surface at time $0.2 \mathrm{~ms}$ was improved by $37.7 \%$ and $31.8 \%$ on structures H5A55_9 and H3A33_25, respectively. Thus we can conclude that when the additional area is kept the same, the higher pillar structure has better solid-liquid heat transport performance than the dense array structure. The limited pillar height of the dense array structure is not beneficial for heat transfer in the 
vertical direction. Because of the relatively higher conductivity of solid material than liquid, higher pillar structure is able to spread heat faster in vertical direction, thus the liquid domain can be heated more uniformly and faster.

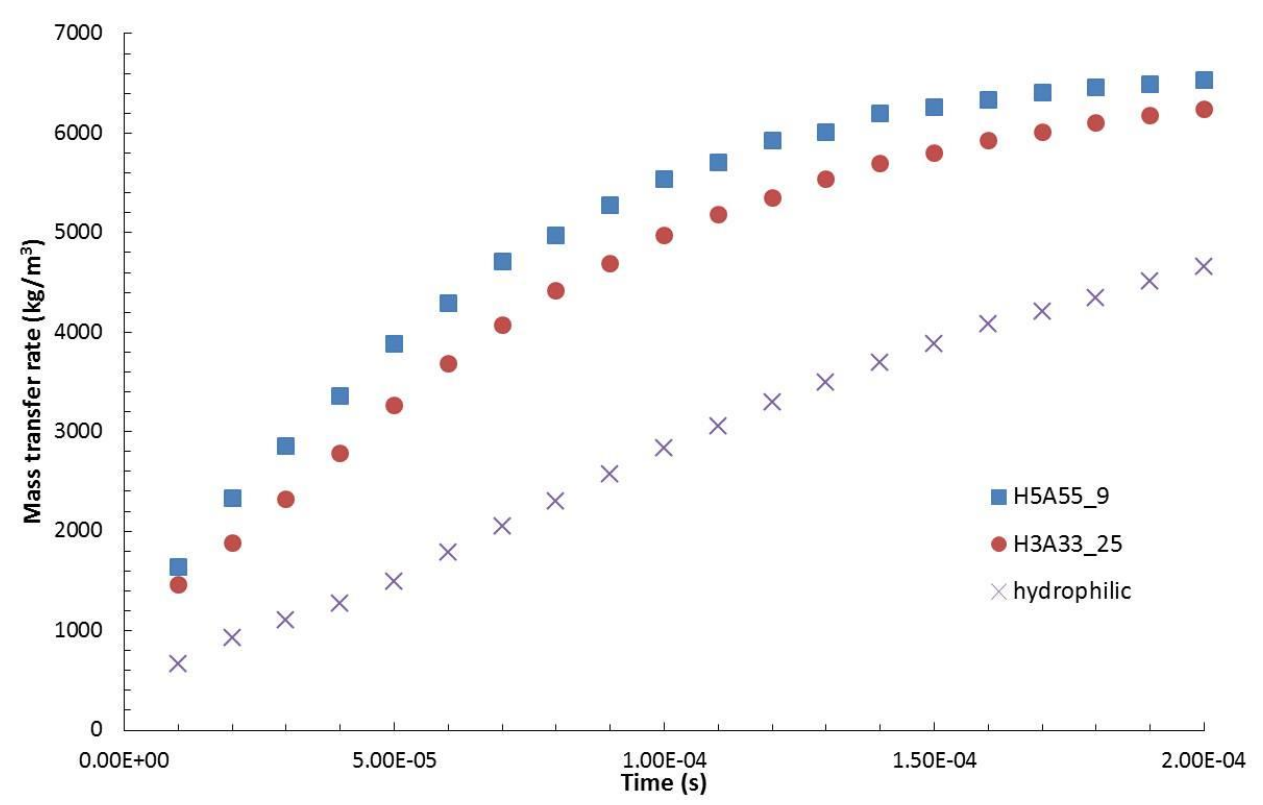

Fig. 24 Mass transfer rate on surfaces for comparison of impact between pillar height and pillar array density $\left(T_{\mathrm{w}-b o t t o m}=400 \mathrm{~K}\right)$

\subsubsection{Constant Heat Flux Boundary Condition}

Fig. 25 demonstrated the temperature contours on surfaces selected to be compared for comprehending the impact of pillar height and pillar array density. For precluding the heat enhancement influence induced by solid-liquid interface area, the structure with dense array H3A33_25 and structure with high pillars H5A55_9 contains the same substrate interfacial area. Except for the relatively lower solid temperature achieved by H5A55_9 at time 0.05ms, the solid temperature of the two structured surface cannot be observed distinctly in Fig. 25. This result indicates that the temperature difference between two structures is less than $1.5 \mathrm{~K}$. 


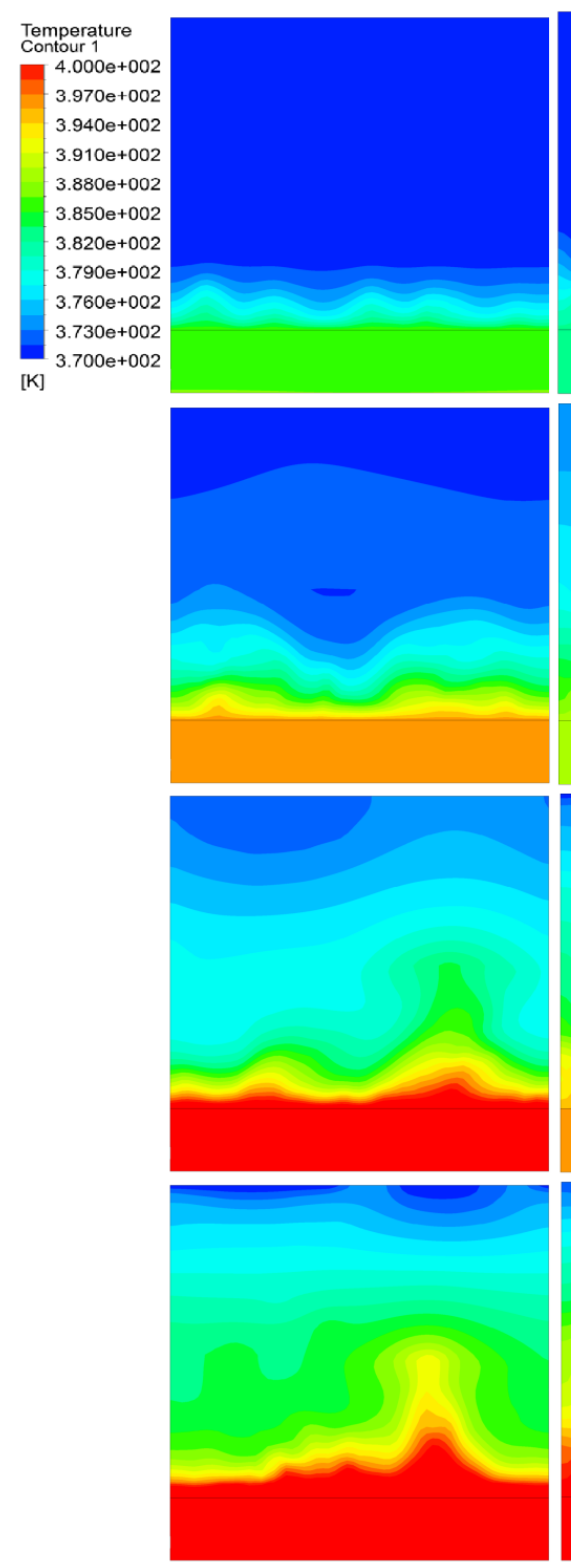

Hydrophilic

Flat Surface
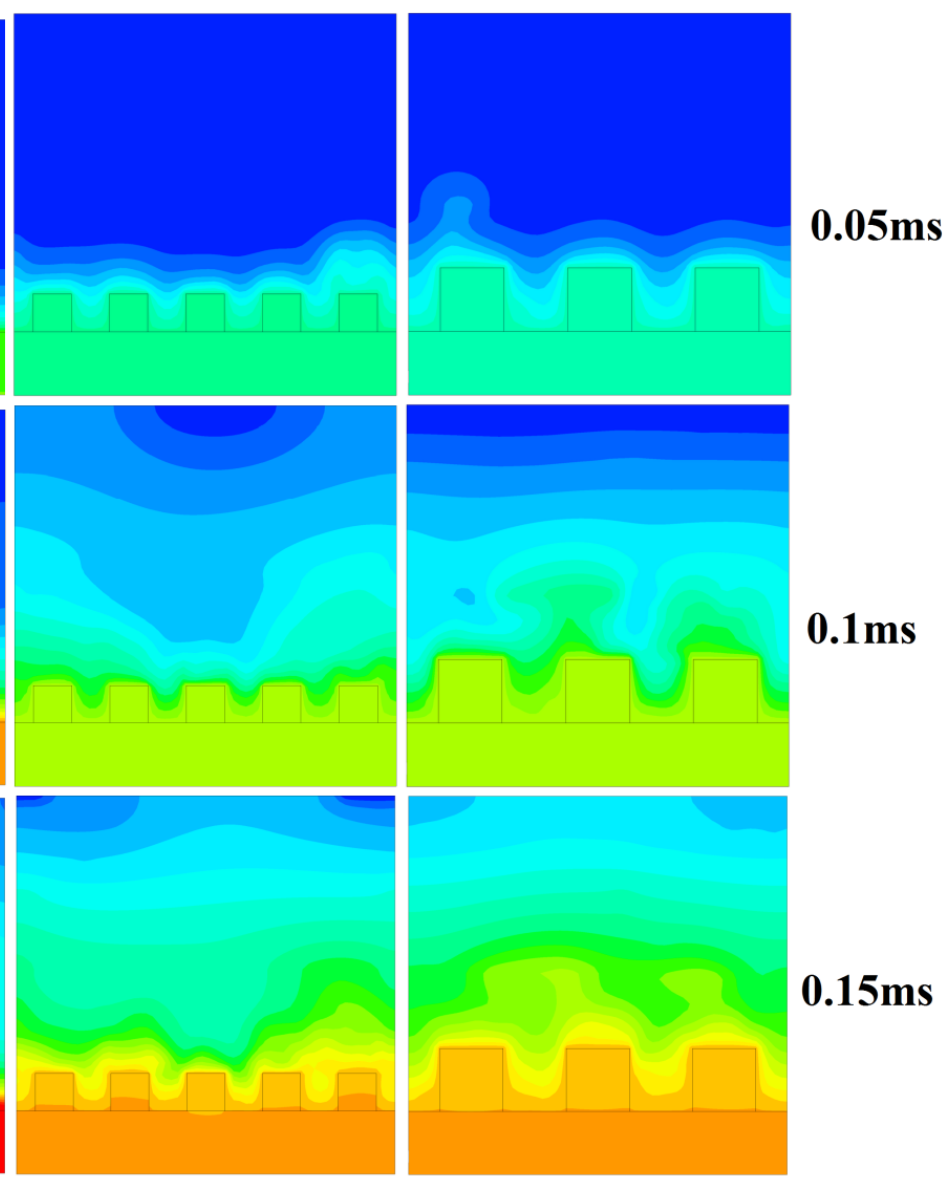

\section{$0.1 \mathrm{~ms}$}

$0.15 \mathrm{~ms}$
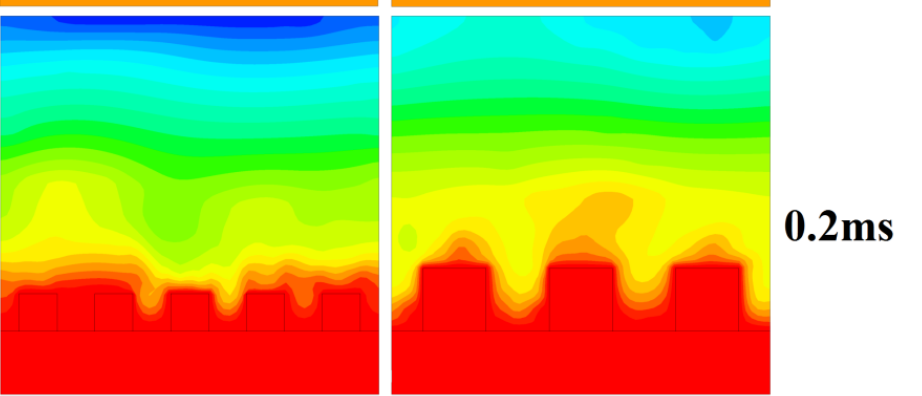

H3A33_25



H5A55_9

Fig. 25 Temperature contours on surfaces for comparison of impact weight

\section{between pillar height and pillar array density}

However, for structure H5A55_9 at time $0.15 \mathrm{~ms}$ and $0.2 \mathrm{~ms}$, the higher temperature region denoted by the yellow and green zones was distributed on a larger scale in fluid domain. 
As described before, stronger mass transfer from liquid to vapor comes with the higher average liquid temperature, and eventually results in better heat dissipation and lower heat source temperature. Hence the relatively lower solid temperature on structure H5A55_9 can be expected beyond $0.2 \mathrm{~ms}$.



Fig. 26 Bottom surface temperature on surfaces for comparison of impact weight between pillar height and pillar array density $\left(q_{w}{ }^{\prime \prime}=700 \mathrm{~W} / \mathrm{cm}^{2}\right)$

Fig. 26 presents the temperature variation of the bottom surface, where the heat source was located with a constant heat flux of $700 \mathrm{~W} / \mathrm{cm}^{2}$. The average bottom surface temperatures during $0.21 \mathrm{~ms}$ of the two structured surfaces are very close. For structure H3A33_25 and H5A55_25, the time averaged temperature is $389.24 \mathrm{~K}$ and $389.10 \mathrm{~K}$. Although the cooling performance of two structures cannot be distinguished from heat source temperature variation, 
the future trend of bottom surface temperature curve can still be predicted by considering the mass transfer rate on those surfaces.

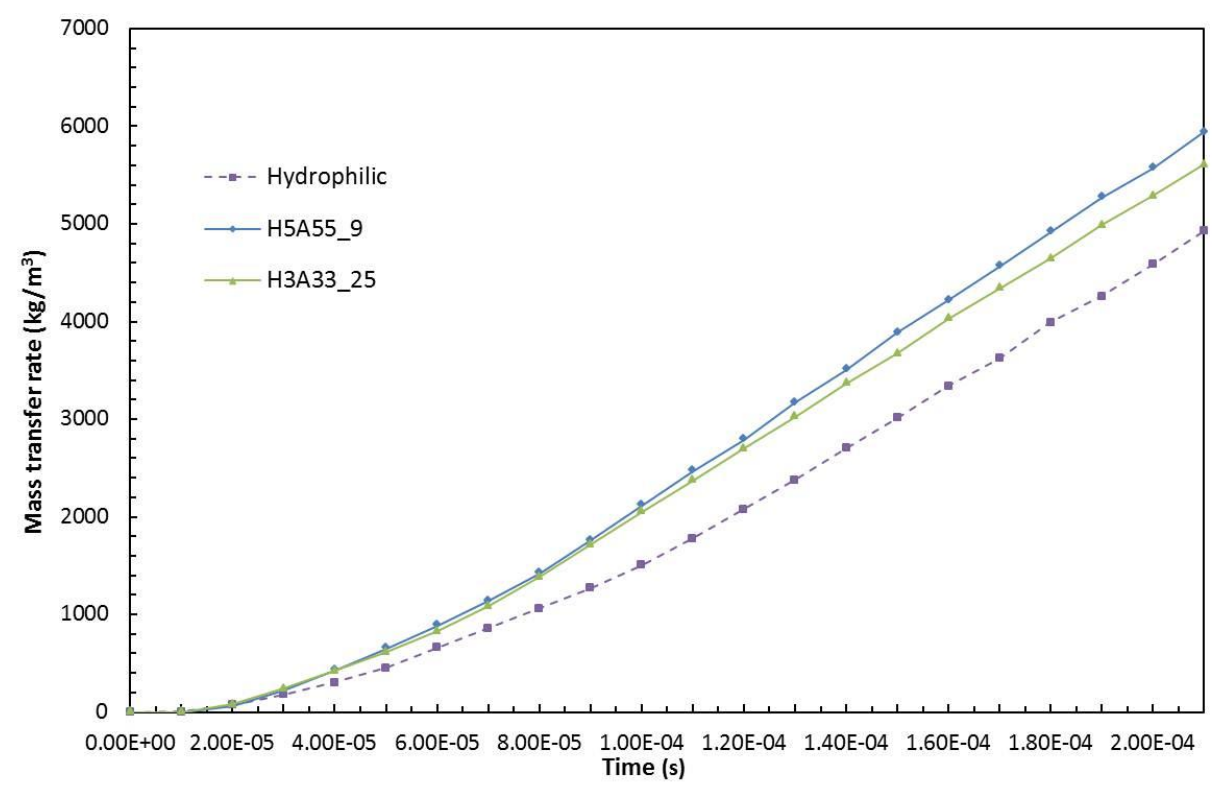

Fig. 27 Mass transfer rate on surfaces for comparison of impact weight between pillar height and pillar array density $\left(q^{\prime \prime}=700 \mathrm{~W} / \mathrm{cm}^{2}\right)$

Fig. 27 indicated the liquid-vapor mass transfer rate variation on different structures. The time averaged liquid-vapor mass transfer rate of H5A55_9 is 4.8\% higher than the rate of H3A33_25. When time comes to $0.21 \mathrm{~ms}$, the liquid-vapor mass transfer rate on structures H3A33_25 and H5A55_9 was increased by $13.7 \%$ and $20.4 \%$, respectively. Higher mass transfer rate achieved on higher pillar structure implies its stronger phase change intensity. Thus thermal energy can be taken away faster on the higher pillar structure and comparatively a lower heat source temperature on it can be expected. 




Fig. 28 Heat transfer coefficient on surfaces for comparison of impact weight between pillar height and pillar array density $\left(q_{w} "=700 \mathrm{~W} / \mathrm{cm}^{2}\right)$

Fig. 28 demonstrates the heat transfer coefficient variation. The time averaged heat transfer coefficient of H5A55_9 is higher than the value of H3A33_25 by 9.7\%. We can therefore obtain the conclusion that the heat dissipation effect of structure H5A55_9 is more prominent than the effect brought by H3A33_25. The factor of pillar height plays a more significant impact on heat dissipation than the factor of pillar array density. In other words, higher pillar structure is more desirable for two-phase heat transfer than dense array structure when flooding, if the pillar array density and pillar height requirement cannot be achieved simultaneously.

The fluctuations of the heat transfer coefficient curve reflect the influence of bubble accumulation and departure on solid-liquid heat transfer. For the reason that bubble thermal resistance is particularly high, the accumulation of bubbles will greatly decrease the heat flux, 
while departure of a large bubble will suddenly increase the heat flux. Thus bubble behavior control and the bubble departure promotion becomes an issue for higher efficiency thermal dissipation. This is expected to be solved by the surface wettability gradient in the pillar height direction. The impact of wettability texture on structured surface will be discussed in the next section.

\subsection{Comparative Studies for Structures with Wettability Texture and Hydrophilic Structure}

The bottom surface temperature variation is displayed by Fig. 29 for structures with different wettability arrangements when the thermal energy input with constant heat flux is $700 \mathrm{~W} / \mathrm{cm}^{2}$. Although the relatively lower bottom surface temperature of the structured surface with engineered wettability (bi-philic structured surface) can be observed in Fig. 29, the temperature difference of the two surfaces is so small that the cooling performance was improved in limited extent by the bi-philic structured surface. The time-averaged bottom surface temperature for bi-philic structured surface is $0.3 \mathrm{~K}$ cooler than the hydrophilic structured surface during the $0.21 \mathrm{~ms}$ period. The existence of the wettability gradient can only reduce the time averaged temperature rise by $1.7 \%$. Similarly, the total temperature rise for the hydrophilic structured surface until time $0.21 \mathrm{~ms}$ is $401.8 \mathrm{~K}$, while the corresponding value for the bi-philic structured surface is $402.3 \mathrm{~K}$. When compared with the total bottom surface temperature rise on hydrophilic structured surface, the enhanced cooling capacity of the bi-philic case is merely $0.5 \mathrm{~K}$, decrease the total temperature rise by $1.7 \%$. 




Fig. 29 Bottom surface temperature on surfaces with different wettability texture $\left(q_{w}{ }^{\prime \prime}=700 W / \mathrm{cm}^{2}\right)$

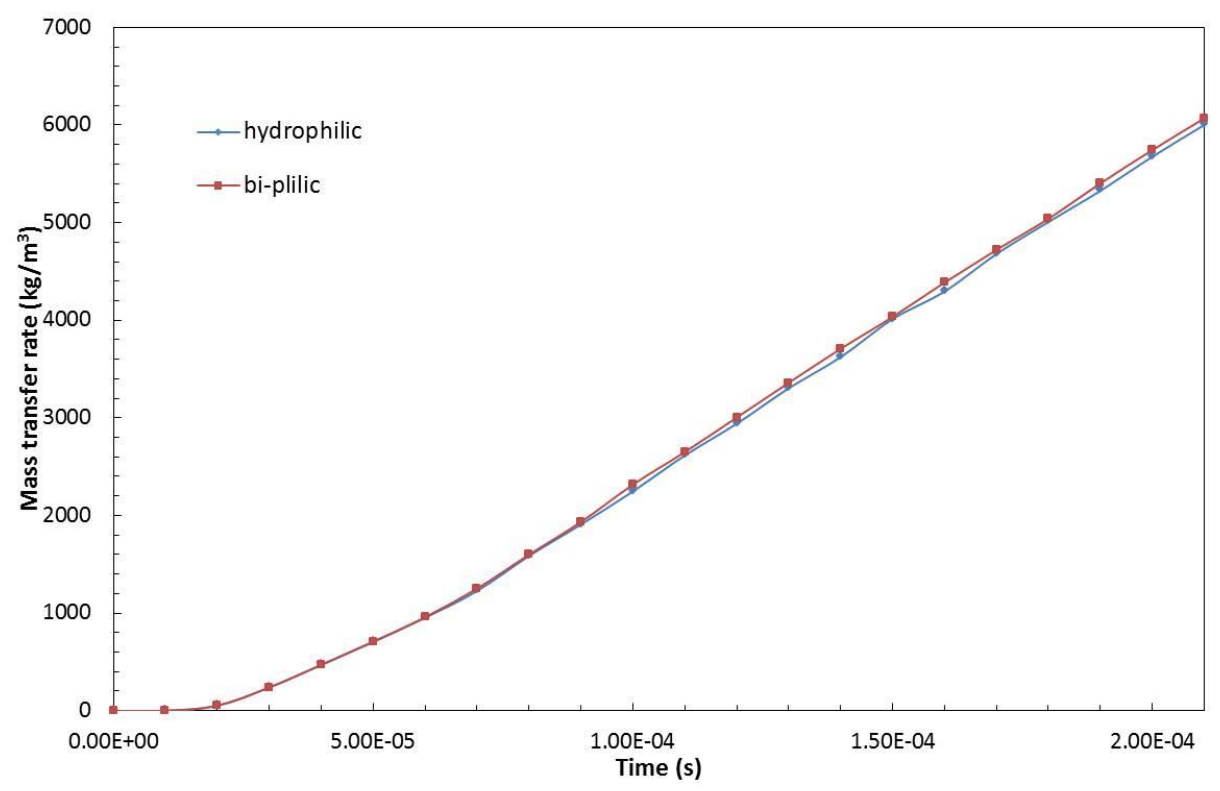

Fig. 30 Mass transfer rate on surfaces with different wettability texture

$$
\left(q_{w}{ }^{\prime \prime}=700 \mathrm{~W} / \mathrm{cm}^{2}\right)
$$


Fig. 30 demonstrates the liquid-vapor phase change intensity upon the hydrophilic structured surface and bi-philic structured surface. The comparatively higher liquid-vapor mass transfer rate on the bi-philic structure affords a reasonable explanation for its better cooling effect, although the improvement is not apparent. Compared with the hydrophilic structured surface, the time-averaged mass transfer rate of the bi-philic structured surface is $1.4 \%$ higher, and the instant mass transfer rate of the bi-philic structured surface at $0.21 \mathrm{~ms}$ is enhanced by only $1.1 \%$.

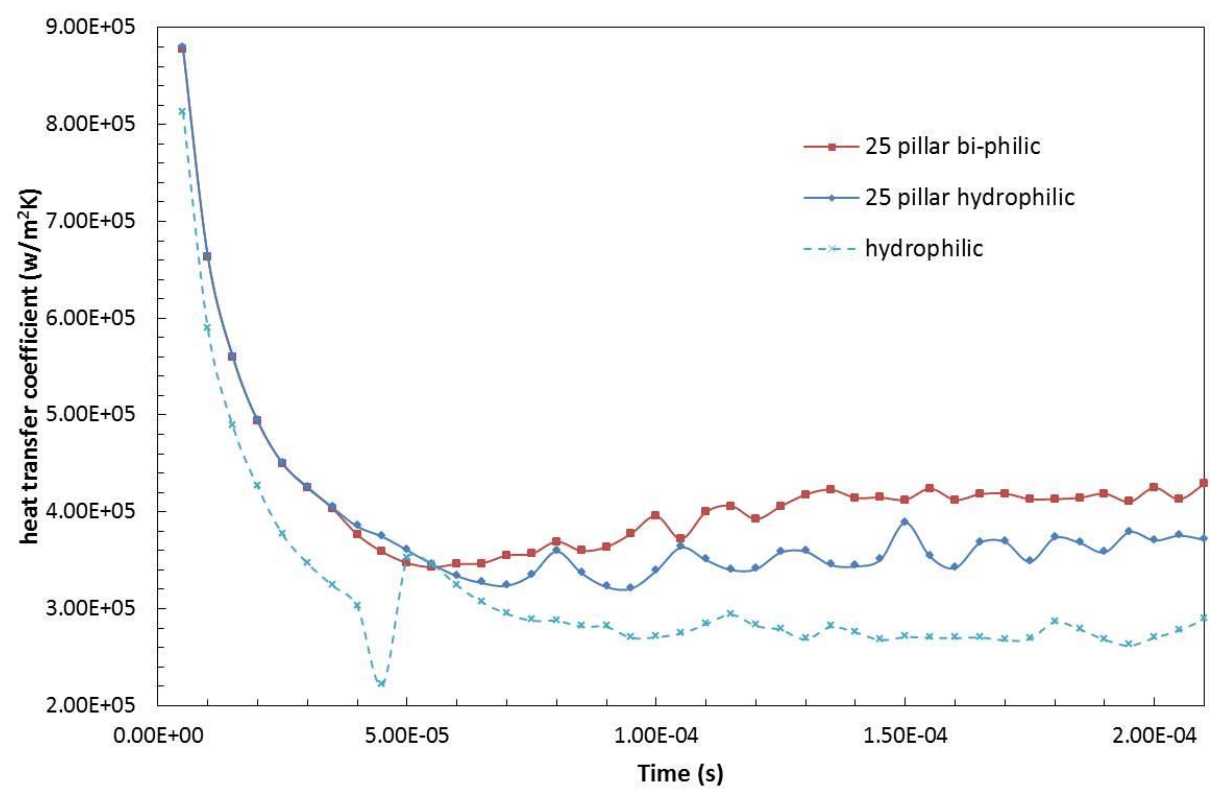

Fig. 31 Heat transfer coefficient on surfaces with different wettability texture $\left(q_{w}{ }^{\prime \prime}=700 W / \mathrm{cm}^{2}\right)$

Fig. 31 depicts the heat transfer coefficient of the structured surface with and without wettability gradient, and respective enhancement based on the heat transfer coefficient of hydrophilic flat surface. Like the former results, the bi-philic structured surface displayed an improvement on cooling performance than the hydrophilic structured surface. Compared with the 
hydrophilic flat surface, the time-averaged heat transfer coefficient improvement by using hydrophilic structured surface is $22.8 \%$, and by using the bi-philic structured surface, it is $33.2 \%$.

From the above data and comparison within the simulation period $0.21 \mathrm{~ms}$, we may find that the cooling performance of the hydrophilic structured surface and bi-philic structured surface is very close. The phase change and cooling enhancement achieved by bi-philic structured surface is in limited extent. The main reason for the limited thermal improvement of the bi-philic structure is caused by the dramatic loss of heat transfer on hydrophilic pillar top surface. The bubble attached on the hydrophobic surface blocked the liquid-solid connection, thereby causing an extreme increase in thermal resistant. However, the hydrophobic pattern is required for providing the source momentum for facilitating bubble departure. Thus the resulting heat transfer performance is determined by a trade-off between the bubble departure improvement within the pillar forest and the bubble pinning at hydrophobic pillar tops. The optimized design for bi-philic structure with less hydrophobic surface area was expected with higher heat transfer efficiency and better cooling performance. 


\section{CHAPTER 5: CONCLUSIONS}

The present paper developed a transient 3-D Volume of Fluid model for investigating the two phase cooling characterisitcs of the enhanced surface, which was modified by the combination of microstructures and engineered wettability, under flooding conditions of dropletbased thermal management. The simulation result confirmed that the bubble motion can be guided upward by the combination of microstructures and engineered wettability. This combination can reduce bubble sizes and enhance bubble departure rates when compared with the flat surfaces and hydrophilic structured surface. When compared to the flat surface, the micro-pillar structured surface with engineered wettability can effectively enhance the solidliquid heat transportation efficiency, liquid-vapor phase change intensity, and further provide a better cooling effect with lower surface temperature attached to the heat source. The structured surface with higher pillars, and structure composed by denser pillar array, enable liquid water to be heated faster and more uniformly, which is desirable for heat dissipation and phase change. The factor of pillar height was validated to play a more significant role than the pillar array density on heat dissipation from solid to liquid when the interfacial areas are the same. The structure with higher pillars is expected to have a better cooling effect than the dense pillar array surface beyond $0.21 \mathrm{~ms}$. This result indicates that for achieving better phase change cooling and higher heat dissipation efficiency when flooding, the micro-pillar fabrication should place emphasis on the factor of pillar height, rather than the pillar array density if the pillar array density and pillar height requriement cannot be achieved simultaneously. For wettability texture

on micro-pillar structure, the resulting heat transfer performance is determined by a trade-off 
between the bubble departure improvement within the pillar forest and the bubble pinning at hydrophobic pillar tops.

Our Future work will focus on investigating the effects of different microstructure topology and dimension on the thermal and dynamic aspects at different levels of dry-out. The cycle from droplet feeding, to flooded regime, and ultimate dry-out regime will be simulated for understanding the boiling and evaporation characteristic in whole process of phase change cooling. 


\section{REFERENCES}

[1] E. S. I. A. E. United States Semiconductor Industry Association (SIA), the Japan Electronics and Information Technology Industries Association (JEITA), the Korean Semiconductor Industry Association (KSIA), and the Taiwan Semiconductor Industry Association (TSIA). International Technology Roadmap for Semiconductors. 2009; Available from: http://www.itrs.net/Links/2009ITRS/Home2009.htm.

[2] T. D. Swanson and G. C. Birur, NASA thermal control technologies for robotic spacecraft. Applied Thermal Engineering, 23(9) (2003).

[3] G. E. Moore, Cramming more components onto integrated circuits. Electronics, 38(8) (1965) 114-117.

[4] A. Bar-Cohen, P. Wang, and E. Rahim, Thermal management of high heat flux nanoelectronic chips. Microgravity Science and Technology, (2007).

[5] I. Mudawar, Assessment of high-heat-flux thermal management schemes. Components and Packaging Technologies, IEEE Transactions on, 24(2) (2001) 122-141.

[6] D. B. Tuckerman and R. F. W. Pease, High-performance heat sinking for VLSI. Electron Device Letters, IEEE, 2(5) (1981) 126-129.

[7] X. F. Peng and G. P. Peterson, Convective heat transfer and flow friction for water flow in microchannel structures. International Journal of Heat and Mass Transfer, 39(12) (1996) 25992608.

[8] T. M. Adams, S. I. Abdel-Khalik, S. M. Jeter, and Z. H. Qureshi, An experimental investigation of single-phase forced convection in microchannels. International Journal of Heat and Mass Transfer, 41(6-7) (1998) 851-857.

[9] W. Qu, G. M. Mala, and D. Li, Heat transfer for water flow in trapezoidal silicon microchannels. International Journal of Heat and Mass Transfer, 43(21) (2000) 3925-3936.

[10] Y. Chen and P. Cheng, Heat transfer and pressure drop in fractal tree-like microchannel nets. International Journal of Heat and Mass Transfer, 45(13) (2002) 2643-2648.

[11] G. Tunc and Y. Bayazitoglu, Heat transfer in rectangular microchannels. International Journal of Heat and Mass Transfer, 45(4) (2002) 765-773.

[12] J. Judy, D. Maynes, and B. W. Webb, Characterization of frictional pressure drop for liquid flows through microchannels. International Journal of Heat and Mass Transfer, 45(17) (2002) 34773489.

[13] W. Qu and I. Mudawar, Experimental and numerical study of pressure drop and heat transfer in a single-phase micro-channel heat sink. International Journal of Heat and Mass Transfer, 45(12) (2002) 2549-2565.

[14] Z.-Y. Guo and Z.-X. Li, Size effect on microscale single-phase flow and heat transfer. International Journal of Heat and Mass Transfer, 46(1) (2003) 149-159.

[15] M. J. Kohl, S. I. Abdel-Khalik, S. M. Jeter, and D. L. Sadowski, An experimental investigation of microchannel flow with internal pressure measurements. International Journal of Heat and Mass Transfer, 48(8) (2005) 1518-1533.

[16] F. J. Hong, P. Cheng, H. Ge, and G. T. Joo, Conjugate heat transfer in fractal-shaped microchannel network heat sink for integrated microelectronic cooling application. International Journal of Heat and Mass Transfer, 50(25-26) (2007) 4986-4998.

[17] B. Agostini, M. Fabbri, J. E. Park, L. Wojtan, J. R. Thome, and B. Michel, State of the art of high heat flux cooling technologies. Heat Transfer Engineering, 28(4) (2007) 258-281. 
[18] V. Singhal, S. V. Garimella, and A. Raman, Microscale pumping technologies for microchannel cooling systems. Applied Mechanics Reviews, 57(3) (2004) 191.

[19] K. A. Estes and I. Mudawar, Comparison of two-phase electronic cooling using free jets and sprays. Journal of Electronic Packaging, 117 (1995) 323-332.

[20] C. C. Hsieh and S. C. Yao, Evaporative heat transfer characteristics of a water spray on microstructured silicon surfaces. International Journal of Heat and Mass Transfer, 49(5-6) (2006) 962974.

[21] J. Shen, C. Graber, J. Liburdy, D. Pence, and V. Narayanan, Simultaneous droplet impingement dynamics and heat transfer on nano-structured surfaces. Experimental Thermal and Fluid Science, 34(4) (2010) 496-503.

[22] J. T. Cheng and C. L. Chen, Active thermal management of on-chip hot spots using EWODdriven droplet microfluidics. Experiments in Fluids, 49(6) (2010) 1349-1357.

[23] J. T. Cheng and C. L. Chen, Adaptive chip cooling using electrowetting on coplanar control electrodes. Nanoscale and Microscale Thermophysical Engineering, 14(2) (2010) 63-74.

[24] P. Paik, V. K. Pamula, and K. Chakrabarty. Thermal effects on droplet transport in digital microfluidics with applications to chip cooling. in Thermal and Thermomechanical Phenomena in Electronic Systems, 2004. ITHERM '04. The Ninth Intersociety Conference on. 2004.

[25] K. Mohseni. Effective cooling of integrated circuits using liquid alloy electrowetting. in Semiconductor Thermal Measurement and Management Symposium, 2005 IEEE Twenty First Annual IEEE. 2005.

[26] E. Baird and K. Mohseni, Digitized Heat Transfer: A New Paradigm for Thermal Management of Compact Micro Systems. Components and Packaging Technologies, IEEE Transactions on, 31(1) (2008) 143-151.

[27] V. Bahadur and S. V. Garimella, Energy minimization-based analysis of electrowetting for microelectronics cooling applications. Microelectronics Journal, 39(7) (2008) 957-965.

[28] N. Kumari and S. V. Garimella, Characterization of the heat transfer accompanying electrowetting or gravity-induced droplet motion. International Journal of Heat and Mass Transfer, 54(17-18) (2011) 4037-4050.

[29] S. R. Annapragada, S. Dash, S. V. Garimella, and J. Y. Murthy, Dynamics of droplet motion under electrowetting actuation. Langmuir, 27(13) (2011) 8198-204.

[30] E. Baird and K. Mohseni, Digitized heat transfer: a new paradigm for thermal management of compact micro systems. IEEE Trans on Compon and Packag Technol, 31(1) (2008).

[31] J. Kim, Spray cooling heat transfer: The state of the art. International Journal of Heat and Fluid Flow, 28(4) (2007) 753-767.

[32] J. Yang, L. C. Chow, and M. R. Pais, Nucleate boiling heat transfer in spray cooling. Journal of Heat Transfer, 118(3) (1996) 668-671.

[33] B. Horacek, K. T. Kiger, and J. Kim, Single nozzle spray cooling heat transfer mechanisms. International Journal of Heat and Mass Transfer, 48(8) (2005) 1425-1438.

[34] H. Bostanci, D. P. Rini, J. P. Kizito, and L. C. Chow, Spray Cooling With Ammonia on Microstructured Surfaces: Performance Enhancement and Hysteresis Effect. Journal of Heat Transfer, 131(7) (2009) 071401.

[35] H. Bostanci, D. P. Rini, J. P. Kizito, V. Singh, S. Seal, and L. C. Chow, High heat flux spray cooling with ammonia: Investigation of enhanced surfaces for CHF. International Journal of Heat and Mass Transfer, 55(13-14) (2012) 3849-3856.

[36] Z. Zhang, J. Li, and P.-X. Jiang, Experimental investigation of spray cooling on flat and enhanced surfaces. Applied Thermal Engineering, 51(1-2) (2013) 102-111. 
[37] J. Li and G. P. Peterson, Microscale heterogeneous boiling on smooth surfaces-from bubble nucleation to bubble dynamics. International Journal of Heat and Mass Transfer, 48(21-22) (2005) 4316-4332.

[38] A. Luke and D.-C. Cheng, High speed video recording of bubble formation with pool boiling. International Journal of Thermal Sciences, 45(3) (2006) 310-320.

[39] X. Frank, N. Dietrich, J. Wu, R. Barraud, and H. Z. Li, Bubble nucleation and growth in fluids. Chemical Engineering Science, 62(24) (2007) 7090-7097.

[40] D. F. Chao, J. M. Sankovic, B. J. Motil, W.-J. Yang, and N. Zhang, BUBBLE DEPARTURE FROM METAL-GRAPHITE COMPOSITE SURFACES AND ITS EFFECTS ON POOL BOILING HEAT TRANSFER. 17(4) (2010) 297-307.

[41] S. Siedel, S. Cioulachtjian, and J. Bonjour, Experimental analysis of bubble growth, departure and interactions during pool boiling on artificial nucleation sites. Experimental Thermal and Fluid Science, 32(8) (2008) 1504-1511.

[42] R. Ranjan, J. Y. Murthy, and S. V. Garimella, A microscale model for thin-film evaporation in capillary wick structures. International Journal of Heat and Mass Transfer, 54(1-3) (2011) 169179.

[43] Z. Yang, X. F. Peng, and P. Ye, Numerical and experimental investigation of two phase flow during boiling in a coiled tube. International Journal of Heat and Mass Transfer, 51(5-6) (2008) 1003-1016.

[44] E. Da Riva and D. Del Col, Numerical Simulation of Laminar Liquid Film Condensation in a Horizontal Circular Minichannel. Journal of Heat Transfer, 134(5) (2012) 051019.

[45] Y. Zhang, A. Faghri, and M. B. Shafii, Capillary Blocking in Forced Convective Condensation in Horizontal Miniature Channels. Journal of Heat Transfer, 123(3) (2000) 501-511.

[46] H. L. Wu, X. F. Peng, P. Ye, and Y. Eric Gong, Simulation of refrigerant flow boiling in serpentine tubes. International Journal of Heat and Mass Transfer, 50(5-6) (2007) 1186-1195.

[47] S. C. K. De Schepper, G. J. Heynderickx, and G. B. Marin, Modeling the evaporation of a hydrocarbon feedstock in the convection section of a steam cracker. Computers \& Chemical Engineering, 33(1) (2009) 122-132.

[48] C. Fang, M. David, A. Rogacs, and K. Goodson, Volume of fluid simulation of boiling two-phase flow in a vapor-venting microchannel. Frontiers in Heat and Mass Transfer, 1(1) (2010).

[49] J. U. Brackbill, D. B. Kothe, and C. Zemach, A Continuum Method for Modeling Surface Tension. Journal of Computational Physics, 100 (1992) 335-354.

[50] W. Nusselt, Die oberflächenkondensation des wasserdampfes. Zeitschrift des Vereines Deutscher Ingenieure, 60(27) (1916) 541-575.

[51] L. Wang and B. Sunden, Numerical simulation of two-phase fluid flow and heat transfer with or without phase change using a volume-of-fluid model, in 2004 ASME International Mechanical Engineering Congress and Exposition, IMECE 20042004, American Society of Mechanical Engineers: Anaheim, CA, United States. pp. 455 - 462.

[52] C. Aghanajafi and K. Hesampour, Heat Transfer Analysis of a Condensate Flow by VOF Method. Journal of Fusion Energy, 25(3-4) (2006) 219-223.

[53] M. Knudsen, The Kinetic Theory of Gases: Some Modern Aspects. 1934, London: Methuen \&.Co. Ltd.

[54] C. Crowe, M. Sommerfeld, and Y. Tsuji, Multiphase flows with droplets and particles. 1998: CRC Press.

[55] W. H. Lee, A pressure iteration scheme for two-phase flow modeling, in Technical Report LAUR1979, Los Alamos Scientific Laboratory: Los Alamos, New Mexico. pp. 408-431. 
[56] J. h. Wei, L. m. Pan, D. q. Chen, H. Zhang, J. j. Xu, and Y. p. Huang, Numerical simulation of bubble behaviors in subcooled flow boiling under swing motion. Nuclear Engineering and Design, 241(8) (2011) 2898-2908.

[57] D. L. Youngs, Time-dependent multi-material flow with large fluid distortion, in Numerical Methods for Fluid Dynamics Conference, K.W. Morton and M.J. Baines, Editors. 1982, Academic Press. pp. 273-285.

[58] S. G. Jennings, The mean free path in air. Journal of Aerosol Science, 19(2) (1988) 159-166. 